\title{
Frequency Dependent Hydraulic Properties Estimated from Oscillatory Pumping Tests in an Unconfined Aquifer
}

\author{
Avinoam Rabinovich ${ }^{\mathrm{a}, *}$, Warren Barrash ${ }^{\mathrm{b}, \mathrm{c}}$, Michael Cardiff ${ }^{\mathrm{d}}$, David L. \\ Hochstetler $^{\mathrm{c}}$, Tania Bakhos ${ }^{\mathrm{c}}$, Gedeon Dagan ${ }^{\mathrm{e}}$, Peter K. Kitanidis ${ }^{\mathrm{c}}$ \\ ${ }^{a}$ Department of Energy Resources Engineering, Stanford University, Stanford, \\ California, USA \\ ${ }^{b}$ Department of Geosciences, Boise State University, Boise, Idaho, USA. \\ ${ }^{c}$ Department of Civil and Environmental Engineering, Stanford University, Stanford, \\ California, USA. \\ ${ }^{d}$ Department of Geoscience, University of Wisconsin-Madison, Madison, Wisconsin, \\ USA. \\ ${ }^{e}$ Faculty of Engineering, School of Mechanical Engineering, Tel-Aviv University, \\ Tel-Aviv, Israel
}

\begin{abstract}
Oscillatory pumping tests were conducted at the Boise Hydrological Research Site. A periodic pressure signal is generated by pumping and injecting water into the aquifer consecutively and the pressure response is recorded at many points around the source. We present and analyze the data from the field test after applying Fourier analysis. We then match the data with a recently derived analytical solution for homogeneous formations to estimate the equivalent aquifer properties: conductivity $K$, specific storage $S_{s}$ and specific yield $S_{y}$. The estimated values are shown to be in agreement with previous estimates conducted at this site. We observe variations in the esti-
\end{abstract}

${ }^{*}$ Corresponding author at: Department of Energy Resources Engineering, Stanford University, Stanford, California 94035, USA. Tel.: +1 650723 1594. Email address: avinoamr@stanford.edu 
mated parameters with different oscillation periods of pumping. The trend of the parameters with changing period is discussed and compared to predictions by existing theory and laboratory experiments dealing with dynamic effective properties. It is shown that the results are qualitatively consistent with recent works on effective properties of formations of spatially variable properties in oscillatory flow. To grasp the impact of heterogeneity, a simple configuration is proposed, helping explain the observed increase in effective conductivity with decreasing period.

Keywords: Periodic pumping, Oscillatory pumping, Field tests, Equivalent properties, Effective properties, Specific yield.

\section{Introduction}

Estimation of aquifer properties is essential in applications such as aquifer management, remediation of contaminants, and oil or gas exploration. $\mathrm{Nu}-$ merous approaches have been reported and tested throughout the years. Pressure-based methods are those for which changes in water pressure associated with aquifer stimulations are measured and the most prominent of these are constant rate pumping and slug tests. Oscillatory or periodic pumping tests are alternative methods involving consecutive periods of pumping and injection resulting in alternating flow. These tests have the following advantages. First, there is no net water extraction from or injection into the aquifer avoiding possible costs and risks associated with handling and treating contaminated water. Second, periodic pumping should cause less contaminant plume movement than a constant-rate pumping test. Third, the oscillating signal of known frequency is separable from changing background pressure. 
In petroleum applications, this means the tests can be conducted without disrupting the production process. Furthermore, testing over a range of frequencies is possible, a widely applied and powerful tool in signal processing (see e.g., Oppenheim et al. (1989), Zhao et al. (2009)). Finally, the zone in which flow is influenced by pumping expands as period increases, allowing gradual exploration of the spatial properties of the aquifer.

In this work we present and analyze results from oscillatory pumping field tests conducted at the Boise Hydrogeophysical Research Site (BHRS) (Barrash et al., 1999). The experiments involve short periods of oscillation between 10-75 seconds. We arrive at large-scale equivalent or effective aquifer properties by matching the field measurements with an analytical solution to a three-dimensional partially penetrating well model of a homogeneous medium (Dagan and Rabinovich, 2014). Our first goal is to evaluate the feasibility of using oscillatory tests for characterizing aquifer properties. Our second goal is to investigate the behavior of equivalent properties, namely conductivity $K$, specific storage $S_{s}$ and specific yield $S_{y}$ with changing period of pumping oscillation. This may have important implications for future use of oscillatory pumping, being a first step towards a more detailed and complex investigation of aquifer properties.

The idea of periodic pumping tests is found in the literature as far back as Kuo et al. (1972) and the related method of pulse pumping even earlier (Johnson et al., 1966). However, few oscillatory pumping field tests have been previously reported. Rasmussen et al. (2003) conducted a field test using a fully penetrating pumping well with a large period of oscillation (12.5 hours). They estimate the aquifer properties using analytical solutions. 
Their results are only for a single pumping frequency with no discussion of frequency dependent properties. A more comprehensive field test was performed in Renner and Messar (2006) on a fractured sandstone bedrock. A wide range of periods (10-5400 seconds) were applied to a fully penetrating pumping well. The aquifer properties were estimated using a simple analytical solution of infinite radial flow. The variation of aquifer properties with pumping period was analyzed, however no detailed physical mechanism explaining the period dependence was suggested. Additional analysis of data from the Renner and Messar (2006) field test was presented in Fokker et al. (2012b) and Fokker et al. (2013). Recently, Becker and Guiltinan (2010) and Guiltinan and Becker (2015) performed periodic slug tests with periods of 1-4 minutes on a water-bearing bedding plane fracture. Parameters were obtained by fitting data with an analytical solution of a fully penetrating pumping well in a confined aquifer. The parameter variation with period was closely examined in this work. Results showed transmissivity decreases while specific storage increases with increasing period and diffusivity varies by more than an order of magnitude across the range of pumping periods tested. The authors suggest this behavior is associated with flow in fractured media and the details of this behavior were considered by them to be an open question that deserves further analysis. Other related, but less relevant, field tests are mentioned in Cardiff and Barrash (2014) and summarized in their Table 1.

Analytical, numerical, and laboratory investigations on oscillatory pumping tests have also been performed. Analytical solutions and theoretical tools are presented in Black and Kipp (1981), Dagan and Rabinovich (2014), 
Cardiff and Barrash (2014) and Hollaender et al. (2002). Numerical methods tested on synthetic data are reported in Ahn and Horne (2010), Fokker and Verga (2011), Fokker et al. (2012a), Cardiff et al. (2013a) and Bakhos et al. (2014). Laboratory experiments on oscillatory flow in rock samples have been previously conducted, e.g., in Song and Renner (2007).

Many pumping tests attempt to predict the aquifer properties by matching field data to a solution assuming homogeneity. We refer to such estimated properties as "equivalent". Our approach uses measured periodic head fuctuations in observation wells to identify aquifer hydraulic properties, which are estimated using the best fit between measured and computed heads over all measurements in space. These equivalent parameters assume spatial homogeneity, but are allowed to vary with period of testing by estimating them for each oscillation period independently. One of the main findings is a change of equivalent properties with period, which can be attributed to spatial heterogeneity. Equivalent properties are also related to effective ones, defined as a ratio between average quantities, for example mean flux and mean head gradient in the case of effective conductivity (e.g., Renard and De Marsily, 1997). Here, we use the term "effective" to refer to such properties, specifically we will discuss effective properties derived stochastically (e.g., Dagan, 1986). This was the topic of recent works (Rabinovich et al., 2013a,b) dealing with the dependence of effective properties on frequency for one-dimensional mean flow and we show by a simple model that the results are qualitatively consistent. However, the quantitative analysis of the impact of heterogeneity is beyond the scope of the present study.

The discussion on effective properties in oscillatory flows is relatively new. 
In Rabinovich et al. (2013a), frequency dependent effective hydraulic properties were derived for a heterogeneous media composed of randomly distributed spherical inclusions, for the mean one-dimensional configuration of a semi-bounded domain, with an oscillatory uniform head applied on the boundary. This approach was extended to a log-normal distribution of permeability in Rabinovich et al. (2013b). In these works the effective properties were considered complex numbers. An important finding is that for large periods the dynamic effective conductivity is real and equal to the steady state property while for small periods it increases with decreasing period. The analysis of the equivalent and effective properties for the more complicated three-dimensional oscillatory well flow is a topic not yet explored in the stochastic context.

Even for analyses using homogeneous aquifer solutions, all of the previous oscillatory pumping field tests did not consider the impact of the presence of a water table and assume a confined aquifer, neglecting specific yield. In most constant rate pumping tests, the specific yield is important and computed assuming instantaneous drainage (Moench, 2004). However, in high frequency oscillatory flow the specific yield does not comply with this assumption. The topic of specific yield in oscillatory flow has been studied both theoretically, e.g., Green and Ampt (1911), Barry et al. (1996) and in laboratory experiments, e.g., Nielsen and Perrochet (2000), Cartwright et al. (2003), Cartwright et al. (2005). Similar to the effective conductivity and specific storage, the effective specific yield is defined as a complex parameter and found to vary substantially with frequency. The real part of the complex specific yield changes from the steady state value (i.e., instantaneous 
drainage) at low frequencies to zero at high frequencies. This is the result of a capillary fringe effect acting to limit the water exchange between the variably saturated zone above the water table and the fully saturated aquifer (Cartwright et al., 2003).

In this work we apply the solution of oscillatory well flow in homogeneous aquifers to the analysis of results from testing data collected at the Boise Hydrogeophysical Research Site (BHRS) and advance the method of oscillatory pumping tests in a number of ways. First, our pumping tests are three dimensional, i.e., pumping is carried out in finite segments of the well as opposed to the previous tests where fully penetrating wells were used. This allows for vertical flow as well as horizontal, thus sampling the media more thoroughly. Second, we use here for the first time the analytical solution from Dagan and Rabinovich (2014), allowing us to model the partially penetrating finite length pumping well. This solution also takes into consideration the water-table effect and allows estimation of the specific yield. The analysis of specific yield for an oscillatory field test has not been previously conducted, as far as we are aware. Third, the large amount of data from previous field tests conducted in the BHRS allows for a detailed comparison with the current results and therefore a more comprehensive evaluation. Finally, we investigate the period dependence of the equivalent properties showing that the equivalent conductivity $K_{e q}$, specific storage $S_{s, e q}$ and specific yield $S_{y, e q}$ vary with period length and the observed general trends are in agreement with existing theory. We also provide a new mechanism by which low period oscillatory flow in heterogeneous media will tend to increase flow in high conductivity regions, thus increasing the equivalent conductivity. 
We note that this work may be considered as part of a larger effort to develop an oscillatory hydraulic tomography (OHT) technique as suggested by Cardiff et al. (2013a). Previously, transient hydraulic tomography (THT) has been suggested by Cardiff and Barrash (2011) and shown to be feasible in Cardiff et al. (2012) and Cardiff et al. (2013b). The analysis presented here could be considered as a first step prior to an OHT analysis of aquifer properties.

The paper is organized as follows. Section 2 describes the field site and experimental setup. In Section 3 we formulate the problem and present the homogeneous solution we utilize. We also discuss the data processing stage and the method for assessing the aquifer properties using the analytical solution. Section 4 presents results and discussion for the head amplitude and phase as well as the estimated aquifer properties. Further discussion on the period dependence of equivalent properties is given in Section 5. In Section 5.1, we present a simple model to explain how the impact of heterogeneity results in a dependence of effective properties upon period of oscillation. A summary and application of previous results concerning the dependence of specific yield on frequency, based on laboratory tests, is given in Section 6 . The paper is concluded with a summary and conclusions in Section 7.

\section{Field site and experimental setup}

\subsection{Field site}

The BHRS is a research wellfield developed in an unconfined aquifer at a gravel bar adjacent to the Boise River about 15-km upstream from downtown Boise, ID, USA. Eighteen 10-cm ID wells arranged in three rings around 
a central well (Fig. 1) were cored and screened through 18-21 m of coarse cobble and sand sediments (Barrash et al., 2006) and completed in a clay layer below the fluvial aquifer. The aquifer extends from the water table which is commonly at an elevation of about $847.5 \mathrm{~m}$ above mean sea level (AMSL) and down to the impervious aquifer bottom located at an elevation of about $828.5 \mathrm{~m}$. In the central part of the wellfield (inset of Fig. 1) the aquifer stratigraphy, based initially on porosity logs and core (Barrash and Clemo, 2002; Barrash and Reboulet, 2004) has been subsequently confirmed and refined with geophysical studies (e.g., Clement and Barrash, 2006; Mwenifumbo et al., 2009; Moret et al., 2006). Here the aquifer consists of four major cobble-dominated units (Units 1-4) overlain by a sand channel (Unit 5) that pinches out in the center of the wellfield and thickens toward the Boise River. Of the major cobble-dominated units, Units 1 and 3 have relatively low porosity (mean 0.17-0.18) and porosity variance; Units 2 and 4 have higher porosity (mean 0.23-0.24) and more variable porosity, lithology, and facies composition (Barrash and Clemo, 2002; Barrash and Reboulet, 2004). Unit 4 in particular includes well-defined subfacies bodies such that the aquifer has at least three hierarchical levels of organization (heterogeneity structure) between such subfacies, units, and the aquifer as a whole (Barrash and Clemo, 2002). Such subfacies, based on outcrop exposures and geophysical logs, have lens-like or patchy shapes with long dimensions commonly smaller than $5 \mathrm{~m}$ and vertical dimension around $1 \mathrm{~m}$.

Relative to other intensely monitored field research sites, the BHRS has relatively low to moderate heterogeneity. Based on a slug test data set for the thirteen central wells, as presented in Barrash and Cardiff (2013) and Cardiff 
et al. (2011), the overall $\log _{10}(K)$ mean is $-3.045 \mathrm{~m} / \mathrm{s}$ (range is -4.192 to -1.80 ) and $\log _{10}(K)$ variance is 0.093 (the commonly used $\ln (\mathrm{K})$ variance is therefore 0.49). $K$ structure in the BHRS aquifer is generally related to the firstorder layering of the porosity-lithology-geophysical stratigraphy but $K$-facies are not as extensive as porosity-lithology-geophysical units, and $K$-facies magnitudes are generally positively correlated with porosity in the lower part of the stratigraphy but negatively correlated with porosity in the upper part of the stratigraphy (Barrash and Cardiff, 2013; Cardiff et al., 2013b). $K$-facies dimensions are generally 3-20 m laterally and 1-4 m , vertically (e.g., Fig. 11 in Barrash and Cardiff (2013) and Fig. 10 in Cardiff et al. (2013b)). A finer scale of $K$ organization is evident from comparison of

profiles of geophysical and $K$ logs respectively, both individually and from multivariate statistical analysis (e.g., Figs. 7 and 9 in Barrash and Cardiff (2013)). They indicate that $K$-subfacies bodies can be identified at scales and positions similar to, and commonly coincident with, smaller-scale porosity facies described above. Overall, geostatistical structure of $K$ at the BHRS can be modeled as an exponential structure having lateral correlation lengths of $\mathrm{x}=5.9 \mathrm{~m}$ and $\mathrm{y}=4.3 \mathrm{~m}(\mathrm{x} / \mathrm{y}$ are parallel/perpendicular to the Boise river) and vertical correlation length of $1.2 \mathrm{~m}$ (Cardiff et al., 2011; Barrash and Cardiff, 2013).

\subsection{Experimental setup}

The experimental setup consists of four wells, as illustrated in Fig. 2. Oscillatory pumping was carried out in Well B3 in a 1-m long isolated zone of diameter 10 centimeters, sealed above and below with 1-m long packers. In the pumping well, heads were measured in the pumping zone and in the 
zones above and below. Oscillatory flow was introduced using an in-well piston design developed for this experiment. A piston at the land surface was moved by an electric motor connected to a crankshaft which converted rotational energy to reciprocal motion (see Fig. 3a). The surface piston (7.62-cm diameter) was then hydraulically connected to the sealed, downhole piston (5.08-cm diameter) below the water table (see Fig. 3b). This down-hole piston thus directly forced water into the formation at the given testing interval during downward piston movement and pulled water out of the formation during upward piston movement. Test frequencies were set manually (see Fig. 3a).

The pumping zone was varied to three different elevations, as shown in Tab. 1, and periods ranged from 9.8 to 73 seconds. Pumping was typically conducted for a duration of 15-35 minutes depending on the imposed period, with at least thirty periods observed. The pump flow rate is largely determined by the total volume of water injected or extracted over half of a period, $Q_{t o t}$ [liters]. This is equivalent to the area of the piston $(A)$ multiplied by the shaft stroke length $(L)$. Our experiments consist of two different stroke lengths as shown in Tab. 1, resulting in two different values of $Q_{t o t}$. Assuming sinusoidal (or equivalently cosine) pumping of discharge $Q_{w}(t)=\left|Q_{w}\right| \sin (2 \pi t / P)$, the amplitude of the discharge is given by

$$
\left|Q_{w}\right|=\frac{\pi}{P} Q_{t o t}=\frac{\pi A L}{P}
$$

where $\mathrm{P}$ is the period of oscillation. The values of $\left|Q_{w}\right|$ for each field test are shown in Tab. 1 where the dependence both on period and stroke length can be seen.

Three observation wells were used, located at lateral distances of 3.57- 
$10.55 \mathrm{~m}$ from the pumping well (see Fig. 2) with observation intervals spaced about $2 \mathrm{~m}$ apart (i.e., 1-m observation zones separated by 1-m long packers). Small-diameter fiber-optic pressure transducers (FISO Technologies, model FOP-MIV-NS-369) with precision of less than $1 \mathrm{~mm}$ were placed in the observation points. The transducers measured pressure at time intervals of $6.2 \times 10^{-4}$ seconds. A larger time step of 0.1 seconds was taken every 30 measurements in order to reduce the large amounts of stored data.

\section{Data analysis}

In this section we describe the procedure for processing the data from the experiment and for parameter estimation.

\subsection{Governing equations}

We consider an aquifer of unbounded horizontal extent in the $x, y$ plane and of finite depth $z_{A}$, with the water table at $z=0$ when undisturbed $\left(-z_{A}<z<0\right)$. A partially penetrating well of radius $r_{w}$ and length $L_{w}$ lies along the $z$ axis at $-z_{w}<z<-z_{w}+L_{w}$. The flow is governed by Darcy's law and the mass conservation equation resulting in

$$
S_{s}(\mathbf{x}) \frac{\partial H(\mathbf{x}, t)}{\partial t}-\nabla[K(\mathbf{x}) \nabla H(\mathbf{x}, t)]=q_{w}(\mathbf{x}, t) \quad, \quad-z_{A} \leq z \leq z_{0}
$$

where $t[s]$ is time, $H[m]$ is head, $K[\mathrm{~m} / \mathrm{s}]$ is conductivity, $S_{s}[1 / m]$ is specific storage, $q_{w}[1 / s]$ is the well term, $\mathbf{x}=(x, y, z)[m]$, the unperturbed water table is at $z=0$ and $z_{0}$ is the water-table elevation. The water-table boundary condition is given by

$$
S_{y} \frac{\partial H}{\partial t}+K \frac{\partial H}{\partial z}-K \nabla H \cdot \nabla H=0 \quad, \quad z=z_{0}
$$


where $S_{y}[-]$ is the specific yield. The boundary condition at the impervious aquifer bottom and far from the well are given by

$$
\begin{gathered}
\frac{\partial H}{\partial z}=0 \quad, \quad z=-z_{A} \\
H \rightarrow 0 \quad, \quad R \rightarrow \infty
\end{gathered}
$$

where $R=\left(x^{2}+y^{2}\right)^{1 / 2}$ is the horizontal coordinate.

The problem is involved because of the nonlinearity of Eq. (3), the spatial variability of $K$, and the well geometry. We therefore adopt a few simplifying assumptions, commonly used in the literature on the topic. First, the free surface condition (Eq. (3)) is linearized (e.g., Dagan, 1967) by neglecting the quadratic term, assuming that the water-table drop is small compared to the aquifer thickness at rest $\left(z_{0}<<z_{A}\right)$. Subsequently, the flow domain becomes $z_{A}<z<0$, and Eq. (3) is posed on $z=0$ rather than $z=z_{0}$. The well is modeled as usual by a sink/source line, that is, it is assumed that $r_{w}<<L_{w}$ (in our field test $r_{w} / L_{w} \simeq 0.1$ ). For the analysis considered in this work, we are interested in a solution for a homogeneous medium and an equivalent specific yield, i.e., $K, S_{s}$ and $S_{y}$ are assumed constant.

In oscillatory pumping the well discharge per length is a harmonic function of time, which is convenient to represent as the real part of a complex variable, i.e.,

$$
Q_{w}(t)=\left|Q_{w}\right| R e\left[\exp \left(\frac{2 \pi i t}{P}\right)\right]
$$

where $\left|Q_{w}\right|$ is positive and constant and $P$ is the period of the oscillations. After a sufficiently long time from the inception of the pumping, the head becomes also harmonic and we consider here this steady periodic regime solely (a discussion of the transient regime is given by Cardiff et al. (2013b) 
and Bakhos et al. (2014)).

Incorporating the simplifications discussed above and substituting $Q_{w}(t)=$ $\left|Q_{w}\right| \exp (2 \pi i t / P), H(\mathbf{x}, t)=\widetilde{H}(\mathbf{x}) \exp (2 \pi i t / P)$ in Eqs. (2)-(4) we arrive at the following set of equations

$$
\begin{gathered}
\frac{2 \pi i}{P} S_{s} \widetilde{H}-K \nabla^{2} \widetilde{H}=-\left|Q_{w}\right|\left[\chi\left(z+D_{w}\right)-\chi\left(z+L_{w}-D_{w}\right)\right] \delta(x) \delta(y), \\
z<0 \\
\frac{2 \pi i S_{y}}{P} \widetilde{H}+K \frac{\partial \widetilde{H}}{\partial z}=0, z=0 \\
\frac{\partial \widetilde{H}}{\partial z}=0 \quad, \quad z=-z_{A} \\
\widetilde{H} \rightarrow 0 \quad, \quad R \rightarrow \infty
\end{gathered}
$$

where $\chi$ is the Heaviside step function and $\delta$ is the Dirac operator. We note that the solution $\widetilde{H}$ is the complex phasor and the actual head is $H=\operatorname{Re}\left[\widetilde{H} e^{2 \pi i t / P}\right]$, not in phase with $Q_{w}$ (Eq. (5)) if $\operatorname{Im}[\widetilde{H}] \neq 0$. For large periods, the problem reduces to a quasi-steady state one, i.e., $\operatorname{Im}[\widetilde{H}]=0$ and $H=\widetilde{H} \cos (2 \pi t / P)$. The solution to Eqs. (6) - (8) is derived in Dagan and Rabinovich (2014) and summarized in Appendix A. We note that simplified solutions to these equations pertaining to an oscillating point source in an infinite medium and an unconfined aquifer are also presented in the appendix (Eqs. (A3) and (A4), respectively).

\subsection{Data processing}

The data acquired from the field tests consists of hydraulic head as a function of time (see Fig. 4a). In order to match the data with the semianalytical solution and also to remove any noise, it is beneficial to apply a 
Fourier analysis to the time series. Fourier analysis is a decomposition of a time series into a sum of harmonic functions, reducing it to amplitude and phase of oscillations at distinct frequencies $(f=1 / P)$.

First, two initial periods of data were removed in order to avoid transient flow associated with the onset of pumping, thus assuring steady periodic flow. Then, the time series was truncated so that the total time is a multiple of the period in order to minimize spectral leakage. The time series is then interpolated to assure equal time increments between samples. Following this process a fast Fourier transform (FFT) is applied resulting in amplitudes and phases for each frequency. Fig. 4b depicts the results of the FFT applied to the time series of a single observation point. It can be seen that there is one dominant frequency $\left(f_{1}\right)$ corresponding to the induced pumping frequency. Additional dominant frequencies, which are multiples of $f_{1}$, also take part in the construction of the time series. These reflect the fact that due to machinery imperfections the signal generated by our pump is not a perfect sinusoid, but rather a sum of harmonics.

In this work we focus our attention on the first dominant frequency, using only a single harmonic to represent the signal thus simplifying computations in the comparison with the semi-analytical solution. However, the theoretical solution used here can be extended to incorporate multiple frequencies, taking advantage of the linearity of Eqs. (6)-(8). We note that apart from the dominant frequencies, all other frequencies in Fig. 4b appear to be significantly lower, thus pointing to low noise. This is not always the case and noise is apparent in some of the data, particularly in very low frequencies, suggesting transient noise of some sort. 
Next, we select only data which appear to be of good quality as determined by the following criteria. The data from the transducer in the pumping zone in Well B3 represents the quality of the pumping signal and therefore is used to asses the quality of the whole test. Two criteria are evaluated for this data. The first is a ratio, $Q_{1}=\left|H_{f_{2}}\right| /\left|H_{f_{1}}\right|$, where $\left|H_{f_{1}}\right|$ and $\left|H_{f_{2}}\right|$ are the head amplitudes of the first and second dominant frequencies, respectively. A large ratio suggests that the additional frequencies may be significant and therefore an analysis using only the first frequency is not appropriate. We use only tests where $Q_{1}<0.5$, i.e., the first frequency amplitude is at least twice that of the second frequency. The second criterion is a measure of how much of the original signal is reproduced by the harmonic of the first frequency alone. This is represented by the ratio between variances and the criterion is as follows

$$
Q_{2}=1-\frac{\operatorname{Var}\left[H_{F}-H_{f_{1}}\right]}{\operatorname{Var}\left[H_{F}\right]}>0.6
$$

where $H_{F}$ is the full time series. Fig. 4 shows a comparison between $H_{F}, H_{f_{1}}$ and $H_{f_{2}}$, where criteria values are $Q_{1}=0.36$ and $Q_{2}=0.89$.

Any time series which displays non-periodic behavior, i.e., no dominant frequencies in the Fourier domain, is not used. In addition, data from observation wells which have dominant periods different than that of the pump signal, as determined by the first frequency of the FFT, are also discarded. Finally, 3 tests with a lower packer pressure were suspected of having leakage and therefore also removed. In total, only 14 tests (see Tab. 1) out of the 47 tests conducted were used for the results that follow. However, this is most likely due to the stringent criteria we have adopted and the use of prototype equipment. The criteria can be loosened if necessary particularly when 
using multi-frequency components of the FFT. In each of the 14 tests used we typically discard only data from 3-4 transducers (usually the same ones, indicating a malfunction) out of the total 25 transducers in the observation wells.

\subsection{Method of parameter estimation}

We now turn to estimate the aquifer equivalent properties, i.e., conductivity $K_{e q}$, specific storage $S_{s, e q}$ and specific yield $S_{y, e q}$. This is achieved by matching solutions of the homogeneous model with data amplitudes and

phases. In the following, we denote $H_{j}^{\text {field }}$ as the head data from observation point $j$ in the field test and $H_{j}^{\text {model }}$ as the solution located at observation point $j$. We note that when comparing the phase, $\phi_{H}$, a reference phase must be defined, presumably the phase at the pump. However, we found that the amplitude and phase measured at the pump are not reliable and lead to unreasonable phase differences with observation wells. This is expected as the transducer is in the pump zone where turbulence may be significant. Here, we will consistently use an observation point in Well B4 as the reference phase for calculating $\phi_{H}$. Our results were found to be sensitive to the choice of the reference phase, however errors were found to be only up to $50 \%$, which is reasonable for this type of analysis. Furthermore the trends remain the same regardless of choice of reference phase.

The first analysis for property estimation is done using the simpler solution for a confined aquifer, Eq. (A4). The phase from Eq. (A4) is only dependent on the diffusivity $K / S_{s}$. We can therefore use $\phi_{H}$ to obtain $K / S_{s}$ 
by a least squares method. We search for $K / S_{s}$ which minimizes

$$
\sum_{j=1}^{N}\left[\phi_{H_{j}^{f i e l d}}-\phi_{H_{j}^{\text {model }}}\right]^{2}
$$

where $\mathrm{N}$ is the total number of observation points. Then, we use the amplitude to find $K$ which minimizes

$$
\sum_{j=1}^{N}\left[\left|H_{j}^{\text {field }}\right|-\left|H_{j}^{\text {model }}\right|\right]^{2}
$$

and consequently obtain $S_{s}$ as well.

Next, we use the full solution of Eq. (A1), incorporating $S_{y}$. Here, the solution for the phase is dependent on two parameters, $S_{s} / S_{y}$ and $S_{y} / K$. We find these by minimizing Eq. (10) and then match the amplitude minimizing Eq. (11) to obtain $K, S_{s}$ and $S_{y}$.

\section{Results and Discussion}

\subsection{Analysis of measurements}

The data processing procedure described in Section 3.2 produces amplitude and phase for each observation point. Observed amplitudes range between 0.1-10 mm. They depend on the period of oscillation $P$ both through Eq. (A1) and through the imposed amplitude $\left|Q_{w}\right|$ as shown in Eq. (1). It can be seen in Eq. (A1) that the dependence of $|H|$ on $\left|Q_{w}\right|$ is linear (we note that a dependence of $S_{y, e f}$ on $\left|Q_{w}\right|$ is suggested in Section 6, however this has a minor effect on $|H|)$. To remove the impact of variations in $\left|Q_{w}\right|$, we consider from here on a non-dimensional head $K_{\text {ref }}|H| /\left|Q_{w}\right|$, where $K_{\text {ref }}$ is taken as $5 \cdot 10^{-4} \mathrm{~m} / \mathrm{s}$ averaging previous field campaign results for conductivity. 
Field test results are illustrated in Fig. 5 for the non-dimensional head amplitude and phase in the three observation boreholes and for three periods of oscillation. In Fig. 5a, it can be seen that the amplitude generally has a maximum near the elevation of the pump $\left(z_{w}=840 \mathrm{~m}\right)$ and it decreases with distance from the pump. The maximum amplitude in Well C4, farthest from the pumping well, is recorded in an observation zone below the pump at an elevation of about $834 \mathrm{~m}$. A possible explanation for this is the impact of heterogeneity. Previous results (see Fig. 9 in Cardiff et al. 2013b) show that in Well C4 a minimum in conductivity occurs at about $834 \mathrm{~m}$ and this may result in increased head there (head increases with decreasing conductivity as seen in Eq. (A1)).

Fig. 5a also shows an increase in amplitude with increasing period. This behavior is fundamental in oscillatory flow, where low period pressure oscillations will attenuate over shorter distances, as observed by Eq. (A3). The head phase shown in Fig. 5b increases with distance from the pumping well in agreement with Eq. (A3). This represents the time lag between the source signal at the pump and the observation wells. It can also be seen that the phase is larger for smaller periods, as low period pressure signals "travel" slower (Eq. (A3)). Overall, the head curves in Fig. 5, display a similar structure, showing consistency between the three different field tests.

Fig. 6 illustrates the dimensionless head amplitude at Well B4, elevation $837.7 \mathrm{~m}$ as a function of period for all field tests. The increase in amplitude with period can be observed consistently. For comparison, the inset shows theoretical results for head amplitude subjected to an oscillating point source in an infinite medium (Eq. (A3)). It clearly demonstrates the same trend 
of change with period (though the amplitude is lower due to the different geometry and impact of heterogeneity). The consistency of our field test measurements can also be evaluated observing Fig. 6. For tests with pump elevation of 838 and $842.5 \mathrm{~m}$, amplitude increases coherently with period, despite tests being conducted non-consecutively, sometimes on completely different days.

The effect of the pump elevation can also be seen in Fig. 6. For $D_{w}=837$ $\mathrm{m}$ (green curves) the observation point is closest to the pump and therefore the amplitudes are largest, while for $D_{w}=842.5 \mathrm{~m}$ (red curves) the observation point is farthest from the pump and the amplitudes are smallest.

\subsection{Parameter estimation}

Parameter estimation is performed matching the measured amplitudes and phases with the analytical solutions of a confined aquifer (Eq. (A4)) and unconfined aquifer (Eq. (A1)), as explained in Section 3.3.

\subsection{1. $K$ and $S_{s}$}

Results for $K_{e q}$ are presented in Fig. 7a. Values range between $2-7 \times 10^{-4}$ $\mathrm{m} / \mathrm{s}$ and are in good agreement with previous estimates, i.e., $K_{e q}=2 \times$ $10^{-4} \mathrm{~m} / \mathrm{s}$ (Cardiff et al., 2013b) and $K_{e q}=8.9 \times 10^{-4} \mathrm{~m} / \mathrm{s}$ (Barrash and Cardiff, 2013). A clear trend is observed in which $K_{e q}$ hardly varies for large $P$ yet increases with decreasing period for small $P$. A similar trend was observed by Becker and Guiltinan (2010) and Guiltinan and Becker (2015). This is characteristic of dynamic effective conductivity (Rabinovich et al., 2013a,b), denoted $K_{e f}$, where the real part of $K_{e f}$ tends to the steady state $K_{e f}$ at low frequencies (large $P$ ) and increases at high frequencies (small $P$ ). 
Further discussion is given in Section 5. Fig. 7b presents results for $S_{s, e q}$. Values range between $0.65-2.4 \times 10^{-5} \mathrm{~m}^{-1}$ for the confined aquifer analysis and $0.25-0.95 \times 10^{-5} \mathrm{~m}^{-1}$ for the unconfined aquifer model (limited to larger periods). Much less work has been conducted at the BHRS on specific storage estimation, yet previous estimates of about $3 \times 10^{-5} \mathrm{~m}^{-1}$ have been reported in Barrash et al. (2006), which is in reasonable agreement with our results. A similar trend with period that was observed for $K_{e q}$ is also seen for $S_{s, e q}$ (further discussion in Section 5).

Altogether, Fig. 7 shows reasonably low variations in parameter estimation between the different tests and a fairly consistent trend (which can be attributed to the impact of heterogeneity). This supports the reliability of the experiment and method. A slight increase in $K_{e q}$ is seen as the pumping zone elevation is raised. This is most likely due to changes in the aquifer properties near the different pumping locations. In fact, previous analyses show that conductivity in the pumping well (B3) increases with elevation in the range of our pumping zones (see Barrash and Cardiff (2013), Fig. 3d, Units $3,4)$, in agreement with the increase of $K_{e q}$ seen here. Fig. 7 also shows that using the unconfined aquifer model does not significantly alter the results, however a small decrease in $K_{e q}$ and $S_{s, e q}$ is seen. This is because allowing flow in the upper boundary of the model acts to reduce head amplitudes leading to lower estimates of $K_{e q}$ and $S_{s, e q}$ (model amplitude increases with decreasing $K$ and $S_{s}$ as observed through Eqs. (A1) and (A3)). Although the unconfined model was seen to have a small contribution in estimating aquifer conductivity and specific storage, it is essential for estimating the specific yield. 


\subsection{2. $S_{y}$}

Results for equivalent specific yield are presented in Fig. 8, for pumping in the highest interval, the one closest to the water table. For pumping tests in lower zones, i.e., $z_{w}=840$ and $z_{w}=837 \mathrm{~m}$, results are not shown since a minimum of Eq. (10) is not obtained as required in the parameter estimation procedure. Values of $S_{y, e q}$ are very low, ranging between $0.9-1.6 \times 10^{-4}$. Previously, values of $S_{y}=0.01-0.06$ were reported by Barrash et al. (2006) for constant rate pumping tests, two orders of magnitude larger than those found here. A similar finding of low $S_{y}$ in periodic pumping is mentioned in Rasmussen et al. (2003). Part of the reason for this difference is that an oscillating water table behaves quite differently than in conventional pumping tests, particularly at short periods. Capillary fringe forces act to limit the moisture exchange between the saturated zone below the water table and the partially saturated zone above. This has been the subject of a number of investigations relying on laboratory experiments, e.g., Nielsen and Perrochet (2000), Cartwright et al. (2003) and Cartwright et al. (2005). They show that $S_{y, e f}$ may decrease by more than two orders of magnitude at small periods of oscillations, compared to the steady state (instantaneous drainage) $S_{y, e f}^{s t}$ prevailing at large periods. The trend observed in Fig. 8 of decreasing

$S_{y, e q}$ with smaller periods is in line with the trends reported in laboratory experiments. Further discussion on the results for $S_{y, e q}$ and its variation with period appears in Section 6. 


\section{Discussion on period dependence of $K$ and $S_{s}$}

For a discussion of period dependence found in our field test results, we turn to consider previous work on this matter. In Renner and Messar (2006), period dependence of properties was hypothesized to be related to a "backbone" of high conductivity surrounded by stagnant "dead end" zones. Later, Cardiff et al. (2013a) showed that different periods of oscillations lead to changes in sensitivity maps. This implies that equivalent properties effectively average over different regions, which in a non-stationary media results in period dependence. We note that both explanations may be valid and Guiltinan and Becker (2015) even suggest that the changes in sensitivity maps may turn out to be a generalization of the "backbone" conceptualization. It would be valuable in the future to evaluate these in view of our field test results, however, this is beyond the scope of the current work.

Here, we will discuss theoretical results of equivalent properties which can give intuition on one hand and may be further developed in the future for quantitative comparisons with field test results. This could be a step towards a quantitative comparison between the impact of sensitivity changes, suggested by Cardiff et al. (2013a), and theoretical results for equivalent properties, in order to determine the mechanism responsible for the period dependence. Our discussions will focus on effective aquifer properties $\left(K_{e f}\right.$, $\left.S_{s, e f}\right)$ in oscillatory flows, derived analytically. We will also present a simple mechanism of $K_{e f}$ variations with period which may shed some light on our field test results.

Effective properties are typically defined by averaging the governing equa- 
tions, i.e. Eq. (2) as

$$
\langle\mathbf{q}\rangle=-K_{e f}\langle\nabla H\rangle, \quad \nabla \cdot\langle\mathbf{q}\rangle=-S_{s, e f}\left\langle\frac{\partial H}{\partial t}\right\rangle
$$

where $\mathbf{q}$ is the specific discharge and \langle\rangle denotes ensemble or spatial averaging. In the case of oscillatory flow, each term in Eq. (12) is represented by an amplitude $|H|$ and phase $\phi_{H}$, for instance $H=|H| \sin \left(2 \pi t / P+\phi_{H}\right)$. Generally, it is not possible to define a single effective property relating between both amplitude and phase. Therefore, we adopt the approach of Rabinovich et al. (2013a,b), defining dynamic effective properties in a complex representation prescribed by Eq. (6) as follows

$$
\begin{array}{r}
\langle\widetilde{\mathbf{q}}\rangle=-K_{e f}\langle\nabla \widetilde{H}\rangle \\
\nabla \cdot\langle\widetilde{\mathbf{q}}\rangle=-\frac{2 \pi i}{P} S_{s, e f}\langle\widetilde{H}\rangle
\end{array}
$$

where $q=\widetilde{q} \exp (2 \pi i t / P)$ and $H=\widetilde{H} \exp (2 \pi i t / P)$. This approach has been widely used on smaller scales, e.g., Smeulders et al. (1992), where pore scale flow is averaged to the Darcy scale.

It is our assumption here that the equivalent properties estimated in our field tests are closely related to the effective properties for one-dimensional flow as far as the dependence on frequency is concerned. We also note that our estimated properties from the field tests are real valued while those defined by Eqs. (13) are complex. In theory, complex equivalent properties can be estimated in our field tests by allowing $K$ and $S_{s}$ to be complex when minimizing Eqs. (10) and (11). However in practice this makes the process of converging to a global minimum much more difficult as the number of unknowns are increased. In this work, we attempted to seek complex equivalent 
properties but were not able to obtain a global minimum for the imaginary part of the parameters. Nevertheless, we found that the real part of the properties converges to a global minimum and values are similar to those shown in Fig. 7. This is in line with results from Rabinovich et al. (2013a) showing that in most cases the real parts of $K_{e f}$ and $S_{s, e f}$ are a good approximation for the effective properties and the imaginary part can be dropped.

Previous work by Rabinovich et al. (2013a) and later by Rabinovich et al. (2013b) derived $K_{\text {ef }}$ for flow in an aquifer with an oscillating boundary. Heterogeneity was represented by spherical inclusions randomly distributed in a dense configuration with conductivities drawn from a log-normal distribution. Specific storage was assumed constant. The conditions under which $K_{\text {ef }}$ was derived are different from those in our pumping tests and a comprehensive comparison cannot be carried out between the two. Nevertheless, in both Rabinovich et al. (2013a) and Rabinovich et al. (2013b) results showed that $K_{\text {ef }}$ always increases as periods of oscillation decrease, in agreement with our field test results and also previous field tests by Guiltinan and Becker (2015).

In Rabinovich et al. (2013a), effective specific storage is also derived. Results show that $S_{s, e f}$ may increase or decrease with smaller periods, depending on the specific storage contrast between spherical inhomogeneities and the background media. In fact, previous field test results by Guiltinan and Becker (2015) show decreasing specific storage with smaller periods as opposed to results in Fig. 7b. This is presumably because their field site heterogeneity is substantially different than in this work, particularly the field site in their experiment consists of a fractured bedrock and pumping is conducted in a target fracture. 


\subsection{Mechanism of period dependence}

In the following we explore a simple case of a spherical inhomogeneity subjected to oscillatory flow in order to draw insight on the period dependence of $K_{e q}$. The mechanism found to be responsible for this behavior may help explain the observed trends in our field experiment.

We consider a single spherical inclusion of radius $R$ and diffusivity $\alpha_{i n}=$ $K_{i n} / S_{s, i n}$ in a semi-infinite medium of diffusivity $\alpha_{0}=K_{0} / S_{s, 0}$, subjected to oscillatory flow (see Fig. 9). The sphere is located at a distance $d$ from a planar boundary of oscillating head with period $P$. Details on this problem and a semi-analytical solution are given in Rabinovich et al. (2012) (see also Rabinovich (2015) for a solution to the two-dimensional case). We define a box region of $L_{b} \times L_{b}$ enclosing the inclusion and proceed by calculating the equivalent conductivity $K_{e q}$ for that box region. The calculation is dependent on the following non-dimensional parameters; $\kappa=K_{\text {in }} / k_{0}, S=S_{s, \text { in }} / S_{s, 0}$, $P^{*}=P \alpha_{e x} /\left(\pi R^{2}\right), d^{*}=d / R$ and $L_{b}^{*}=L_{b} / R$. Results for $K_{e q}$ are shown in Fig. 10a where the general trend with period is seen to be the same as in the field test results (Fig. 7a) and as $K_{\text {ef }}$ from Rabinovich et al. (2013b).

Fig. 10 depicts an example case with parameters $\kappa=0.01, S=1, d^{*}=3$ and $L_{b}^{*}=4$. The complex equivalent conductivity depicted in Fig. 10a is defined by Eq. (13a). The average gradient in the $z$ direction is calculated as follows

$$
\left\langle\frac{\partial \widetilde{H}}{\partial z}\right\rangle=\frac{1}{L_{b}^{2}} \int_{-L_{b}}^{L_{b}} \int_{-L_{b}}^{L_{b}} \frac{\partial \widetilde{H}}{\partial z} d x d z .
$$

The problem is axisymetrical around the $\mathrm{z}$ axis and therefore integration in the $y$ direction is unnecessary and average gradients in the $x$ and $y$ directions are zero. The gradient can also be written as a sum of the averages in the 
interior and exterior of the sphere. i.e.

$$
\left\langle\frac{\partial \widetilde{H}}{\partial z}\right\rangle=\left\langle\frac{\partial \widetilde{H}}{\partial z}\right\rangle_{e x}+\left\langle\frac{\partial \widetilde{H}}{\partial z}\right\rangle_{i n}=J_{e x}+J_{i n}
$$

where the average gradient is denoted $J$. The average flux can be written as

$$
\langle\widetilde{\mathbf{q}}\rangle=K_{e x}\left\langle\frac{\partial \widetilde{H}}{\partial z}\right\rangle_{e x}+K_{i n}\left\langle\frac{\partial \widetilde{H}}{\partial z}\right\rangle_{i n}=K_{e x} J_{e x}+K_{i n} J_{i n}
$$

and by Eq. (13a) the equivalent conductivity follows as

$$
K_{e q}=\frac{K_{0} J_{e x}+K_{i n} J_{i n}}{J_{e x}+J_{i n}} .
$$

Let us consider $K_{e q}^{1}$ and $K_{e q}^{2}$ corresponding to two different periods, $P_{1}^{*}$ and $P_{2}^{*}$ where $P_{1}^{*}>P_{2}^{*}$ and keep all other parameters the same. We seek the conditions for which

$$
\frac{K_{0} J_{e x}^{1}+K_{i n} J_{i n}^{1}}{J_{e x}^{1}+J_{i n}^{1}}<\frac{K_{0} J_{e x}^{2}+K_{i n} J_{i n}^{2}}{J_{e x}^{2}+J_{i n}^{2}}
$$

where we neglect the imaginary parts of $J_{i n}^{1}, J_{i n}^{2}, J_{e x}^{1}, J_{e x}^{2}$. After some basic algebra the solution for the above inequality is given by

$$
\begin{aligned}
& K_{i n}<K_{0} \\
& \frac{J_{e x}^{2}}{J_{e x}^{1}}>\frac{J_{i n}^{2}}{J_{i n}^{1}}
\end{aligned}
$$

where we assume $J_{i n}^{1}, J_{i n}^{2}, J_{e x}^{1}, J_{e x}^{2}$ are positive as they were found to be in the example in Fig. 10. The condition in Eq. (19a) indicates that the increase in $K_{e q}$ is associated with low conducting inhomogeneities as was shown in Rabinovich et al. (2013b). The condition in Eq. (19b) suggests that when decreasing the period, the increase (decrease) in gradient outside of the sphere is larger (smaller) than inside the sphere. This seems to be the important contributing factor for the increase of $K_{e q}$. 
Fig. 10b illustrates $C=\left(J_{i n}^{2} / J_{i n}^{1}\right) /\left(J_{e x}^{2} / J_{e x}^{1}\right)$ for an example case. It can be seen that $C$ at large $P^{*}$ converges to unity, i.e., the exterior and interior gradients change by the same factor and $K_{e q}$ remains unchanged (Fig. 10a). This is typical of a slowly varying or quasi-steady state in which the mean flow is uniform in the vicinity of the sphere (see Rabinovich et al. (2012) for more on the quasi-steady state). For small $P^{*}$ we see that $C$ decreases, dropping below unity, suggesting exterior gradient increases more (or decreases less) than the interior. This occurs when the mean flow near the sphere is nonuniform (varies exponentially in this case) and the exterior diffusivity is much larger than the interior. It indicates that the flow prefers the highly conducting media outside of the sphere and thus overall, conductivity is increased. We note that this mechanism may be somewhat similar to the "backbone" mechanism suggested by Renner and Messar (2006), although they focus on the period dependence of the storage rather than the conductivity.

\section{Comparison of the findings for $S_{y, e q}$ with previous laboratory based results}

The estimation of specific yield is a topic of ongoing controversy in the literature. Despite recent research, e.g., by Mishra et al. (2013), Mathias and Butler (2006) and Tartakovsky and Neuman (2007), the impact of drainage from the unsaturated zone is still not fully understood, even in constant rate pumping tests. For field scale oscillatory pumping tests, there is hardly any record of $S_{y}$ estimation. We therefore discuss our results in comparison to previous theoretical and laboratory work, with hopes of initiating future work dedicated to this issue. 
The impact of the water-table fluctuations is taken into consideration in our analysis through Eq. (7). The specific yield in Eq. (7) is an effective property, i.e., it is assumed to be a property of the medium, independent of time and location. Such an approach is common in modeling constant rate pumping tests, where $S_{y}$ is usually obtained by assuming instantaneous drainage to arrive at $S_{y, \text { ef }}^{s t}$. In the case of oscillatory flow, we allow $S_{y, e f}$ to be a function of period, deviating from $S_{y, \text { ef }}^{s t}$. Investigations of $S_{y, e f}$ in oscillatory flow have been conducted both in theory and experiment. A capillary fringe effect has been found to play a significant role in determining $S_{y, e f}$. Here, we follow the approach of Cartwright et al. (2005) and compare our field test results with their experimental results.

The effective specific yield is defined as

$$
S_{y, e f}^{s t} \frac{\partial h_{t o t}}{\partial t}=S_{y, e f} \frac{\partial h}{\partial t}
$$

where $h$ is the water-table height and $h_{t o t}$ is the equivalent saturated height of the total moisture. $S_{y, \text { ef }}$ is generally complex as fluctuations in the equivalent saturated height are both damped and lagged relative to those of the water table. In Cartwright et al. (2005), experiments on a sand column are conducted to find $S_{y, \text { ef }}$ as a function of period as follows

$$
S_{y, e f}=\frac{S_{y, e f}^{s t}}{1+2.5 i(\widehat{P})^{-2 / 3}}
$$

where

$$
\widehat{P}=\frac{T K}{2 \pi S_{y, e f}^{s t} H_{\psi}} .
$$

Here, $H_{\psi}$ is the equivalent steady saturated height of the the capillary fringe computed from the measured first drying curves by

$$
H_{\psi}=\int_{h}^{\infty} \frac{\theta-\theta_{r}}{\theta_{s}-\theta_{r}} d \psi=\int_{h}^{\infty} \frac{1}{\left(1+|\alpha \psi|^{n}\right)^{m}} d \psi
$$


where $\theta, \theta_{s}$ and $\theta_{r}$ are the local, saturated and residual moisture contents respectively, $\psi$ is the suction head, $m=1-1 / n$ and $\alpha\left[m^{-1}\right]$ and $n$ are empirical parameters of the van Genuchten-Mualem relationship. An alternative to Eq. (21) is an analytical expression derived using the Green and Ampt (1911) approximation give by

$$
S_{y, e f}=\frac{S_{y, e f}^{s t}}{1+i \widehat{P}^{-1}} .
$$

To compare the above to our field test results for $S_{y, e q}$, we must assess the parameters in Eq. (22). While $K$ and $H_{\psi}$ are obtained fairly easily in the experiments of Cartwright et al. (2005), they are difficult to predict in our field tests. The conductivity $K$ should represent the region near the water table and this may deviate significantly from values of $K_{e q} \simeq 5 \cdot 10^{-4} \mathrm{~m} / \mathrm{s}$ discussed previously. $H_{\psi}$ is obtained via Eq. (23), using parameters $\alpha$ and $n$ from Thoma et al. (2014). The range of $\alpha$ and $n$ for the BHRS given by Thoma et al. (2014) is wide and the comparison we wish to hold may change significantly. Therefore, in the analysis below we consider a range of parameters. A value of $S_{y, e f}^{s t}=0.02$ from short-duration pumping tests at the BHRS (Barrash et al., 2006) is taken consistently in Eq. (22).

In Fig. 11 we present a comparison between our field test results for $S_{y, e q}$ (Fig. 8) and previous results given by Eqs. (21) and (24). The black curve is obtained using representative values of $\alpha=8.5 \mathrm{~m}^{-1}, n=2.567$ and $K=1.33 \cdot 10^{-3} \mathrm{~m} / \mathrm{s}$ from Thoma et al. (2014) (Tab. 3, M1). This leads to $H_{\psi}=0.294 \mathrm{~m}$ and a ratio of $K / H_{\psi}=0.0045 \mathrm{~s}^{-1}$. It can be seen that the field test $S_{y, e q}$ is significantly lower than that suggested by both theoretical and empirical curves. However, the trend is in fairly good agreement. The dashed curves show field test results with $K / H_{\psi}$ chosen to optimize fit to the 
theoretical curve for trend (red dashed curve) and $S_{y}$ value (green dashed curve). The optimized fit for trend is obtained by matching slopes of linear interpolations. The curve optimized for trend results in $K / H_{\psi}=0.0072 \mathrm{~s}^{-1}$ and is seen to be fairly close to the representative parameters (black curve). The fit for values of $S_{y}$ is obtained at $K / H_{\psi}=4 \cdot 10^{-4} s^{-1}$ and the trend does not fully agree.

We note that all values of $\widehat{P}$ considered here by taking $4 \cdot 10^{-4}<K / H_{\psi}<$ $0.0072 s^{-1}$ are possible considering reasonable field site parameters. For the representative values (black curve in Fig. 11), the disagreement of $S_{y, \text { ef }}$ values with theory is yet to be explained. Factors other than the capillary fringe effect may be responsible, for instance, the magnitude of water-table oscillations may impact $S_{y, e f}$. A small decrease in $S_{y, e f}$ with smaller water-table fluctuations has been previously shown in Cartwright et al. (2005). More evidence of very low $S_{y, e q}$ in oscillatory pumping is also presented in Rasmussen et al. (2003), where the pumping periods considered were a hundred times larger than here. This also suggests that the cause is not period related. The above discussion is inconclusive and further investigation on this matter with additional field test data is warranted.

\section{Summary and conclusions}

In this work we present results from periodic pumping field tests. First, we apply a procedure for data processing using a Fast Fourier Transform. Then, a semi-analytical solution for three-dimensional flow in an unconfined and homogeneous aquifer subjected to oscillatory pumping is used to estimate the aquifer properties: conductivity $K$, specific storage $S_{s}$ and specific yield 
$S_{y}$. Equivalent conductivity $K_{e q}$ and specific storage $S_{s, e q}$ are estimated and found to be in good agreement with previous results from this test site. The results presented here indicate that oscillatory pumping tests may be used as an alternative to conventional pumping tests. This is encouraging in view of the possibility of using oscillatory hydraulic tomography to estimate aquifer heterogeneity, for which the present simple approach can serve as a first step. Work on heterogeneity estimation using this method with the current data is being conducted at this time.

We also investigate the dependence of equivalent properties on the period of oscillation. We find that $K_{e q}$ and $S_{s, e q}$ increase with smaller period and seem to have small variations at large periods. Similar behavior has been found in effective properties of random media subjected to oscillatory flow. Future derivations of effective properties for conditions resembling those of these field tests will allow for a more detailed comparison. To provide a simple explanation, we present in Section 5.1 calculations for effective conductivity in a very simple model of a single spherical inhomogeneity near an oscillating head boundary. Results show a similar trend of increasing effective conductivity with smaller period. This "toy" model reveals a mechanism by which (in conditions of a non-linear mean flow in the vicinity of an inhomogeneity of low conductivity) raising the period of oscillation will increase flow in the ambient media more than in the inhomogeneity (or decrease flow in the ambient media less than in the inhomogeneity). As a result of this tendency for flow to prefer the highly conducting media and to avoid the low conductivity inhomogeneity, the effective conductivity increases.

Finally, the impact of pumping period on the effective specific yield is 
considered. From analysis of our field tests, we find that values of $S_{y}$ are much lower than those in constant rate pumping tests and they decrease with decreasing period. The trend of change in $S_{y}$ with period is shown to be in reasonable agreement with theoretical results based on laboratory experiments (Section 6). This trend is a result of a capillary fringe effect acting to limit the exchange of fluids between the saturated region below the water table and the partially saturated region above. However, it appears that the low values of $S_{y}$ from our field test cannot be fully explained by this effect. While we suggest a possible impact of the magnitude of the watertable disturbance, further investigation is necessary on this matter. We note that low values of $S_{y}$, as observed in this field test, may imply that the water-table movement can be neglected, reducing the problem to one of a confined aquifer. This may simplify problems incorporating heterogeneity, saving computational time.

The study of parameter variation with period of pumping is important due to implications on future pumping tests and also because of the physical insight gained by understanding the mechanisms responsible for these variations. Future studies should focus on $S_{y}$ in oscillatory pumping and its variations with period. Furthermore, deriving additional $K_{e f}$ and $S_{s, e f}$ for realistic field conditions will improve understanding and estimation of these properties. Finally, heterogeneity estimation using oscillatory hydraulic tomography can be developed. 


\section{Acknowledgments}

This research was supported by Grant No 2012253 from the United StatesIsrael Binational Science Foundation (BSF). Field experimentation was supported by NSF Awards 1215746 and 1215768. Development of the oscillating signal generator used in field experiments for this study was supported by ARO URISP award W911NF1110291 and by cost share with Mt. Sopris Instruments including design input (especially from James Koerlin).

\section{Appendix A: Homogeneous Aquifer Solution}

A semi-analytical solution to Eqs. (6) - (8) is derived in Dagan and Rabinovich (2014) (for details on derivation see their Chapters 3.1 and 4). The exact solution is given by

$$
\begin{array}{r}
H(R, z)=\frac{\left|Q_{w}\right|}{K} \int_{-D_{w}}^{-D_{w}+L_{w}}\left[G_{\infty}\left(R, z-z^{\prime}\right)+G_{\infty}\left(R, z+z^{\prime}\right)\right] d z^{\prime}+ \\
\frac{\left|Q_{w}\right|}{K} \widetilde{g}(R, z)
\end{array}
$$

where

$$
\begin{aligned}
& \tilde{g}(R, z)=\int_{0}^{\infty}\left[-\frac{\left.\exp (\beta z)\left[\exp \left(\beta\left(L_{w}-D_{w}\right)\right)\right]-\exp \left(-\beta D_{w}\right)\right)}{2 \pi \beta A}+\right. \\
& \begin{aligned}
\frac{\exp \left(-2 \beta z_{A}\right)}{2 \pi \beta} \cdot \frac{B \exp (\beta z)-A \exp (-\beta z)}{A+B e^{-2 \beta z_{A}}} \cdot\left\{\frac{\exp \left[\beta\left(L_{w}-D_{w}\right)\right]-\exp \left(-\beta D_{w}\right)}{A}\right. \\
\left.\left.\quad-\frac{\cosh \left[\beta\left(L_{w}-D_{w}\right)\right]}{\beta}+\frac{\cosh \left(\beta D_{w}\right)}{\beta}\right\}\right] p J_{0}(p R) d p . \quad \text { (A2) }
\end{aligned}
\end{aligned}
$$

Here, $\beta=\sqrt{2 \pi i S_{s} /(K T)+p^{2}}, A=2 \pi i S_{y} / P+\beta, B=2 \pi i S_{y} / P-\beta$ and $J_{0}$ is the order zero Bessel function of the first kind. The term $G_{\infty}\left(R, z-z^{\prime}\right)$ in 
Eq. (A1) represents an oscillating point source in an infinite medium located at $R=0, z=z^{\prime}$ (Carslaw and Jaeger (1959) Eq. 10.4.12) and is given by

$$
\begin{gathered}
G_{\infty}\left(R, z-z^{\prime}\right)=-\frac{1}{4 \pi r} \exp (-\gamma r) \quad, \quad \gamma=\frac{\pi S_{s}(i+1)}{K T} \\
\left|G_{\infty}\right|=\frac{1}{4 \pi r} \exp \left(-\frac{\pi S_{s} r}{K T}\right)
\end{gathered}
$$

where $r=\sqrt{R^{2}+\left(z-z^{\prime}\right)^{2}}$. This solution reduces to the Laplace equation Green's function for $S_{s}=0$. If the impact of the water table is negligible, i.e., $S_{y}=0$, solution (A1) reduces to that of a confined aquifer, which is given in an alternative form by expanding the integral in Eq. (A2) to obtain a series of images (see, e.g., Dagan and Lessoff (2011)), as follows

$$
\begin{array}{r}
H(R, z)=\frac{\left|Q_{w}\right|}{K} \int_{-D_{w}}^{-D_{w}+L_{w}}\left[\sum _ { m = 1 } ^ { \infty } \sum _ { n = 1 } ^ { 2 } \sum _ { l = 1 } ^ { 2 } G _ { \infty } \left(R, z+(-1)^{n} z^{\prime}+\right.\right. \\
\left.\left.(-1)^{l} \cdot 2 m D\right)\right] d z^{\prime}
\end{array}
$$

Here, the term $G_{\infty}$ is defined by $G_{\infty}(R, Z)=\exp \left[-\gamma \sqrt{R^{2}+Z^{2}}\right] /\left(4 \pi \sqrt{R^{2}+Z^{2}}\right)$ for any variable $Z$. In our calculations the number of terms taken in the series in Eq. A4 is chosen such that convergence is achieved.

\section{References}

Ahn, S., Horne, R. N., 2010. Estimating permeability distributions from pressure pulse testing. In: SPE Annual Technical Conference and Exhibition. Society of Petroleum Engineers.

Bakhos, T., Cardiff, M., Barrash, W., Kitanidis, P. K., 2014. Data processing for oscillatory pumping tests. Journal of Hydrology 511, 310-319. 
Barrash, W., Cardiff, M., 2013. Hydraulic conductivity distribution from multi-level slug tests and multivariate facies associations in a conglomeratic fluvial aquifer, Boise Hydrogeophysical Research Site. Boise Hydrogeophysical Research Site: Technical Report BSU CGISS, 13-03.

Barrash, W., Clemo, T., 2002. Hierarchical geostatistics and multifacies systems: Boise Hydrogeophysical Research Site, Boise, Idaho. Water Resources Research 38 (10), 14-1.

Barrash, W., Clemo, T., Fox, J. J., Johnson, T. C., 2006. Field, laboratory, and modeling investigation of the skin effect at wells with slotted casing, Boise Hydrogeophysical Research Site. Journal of Hydrology 326 (1), 181198.

Barrash, W., Clemo, T., Knoll, M. D., 1999. Boise hydrogeophysical research site (bhrs): objectives, design, initial geostatistical results. In: Proceedings of the Symposium on the Application of Geophysics to Engineering and Environmental Problems. pp. 389-398.

Barrash, W., Reboulet, E. C., 2004. Significance of porosity for stratigraphy and textural composition in subsurface, coarse fluvial deposits: Boise Hydrogeophysical Research Site. Geological Society of America Bulletin 116 (9-10), 1059-1073.

Barry, D., Barry, S., Parlange, J.-Y., 1996. Capillarity correction to periodic solutions of the shallow flow approximation. Mixing in Estuaries and Coastal Seas, 496-510. 
Becker, M. W., Guiltinan, E., 2010. Cross-hole periodic hydraulic testing of inter-well connectivity. In: Proceedings Thirty-Fifth Workshop on Geothermal Reservoir Engineering.

Black, J., Kipp, K., 1981. Determination of hydrogeological parameters using sinusoidal pressure tests: a theoretical appraisal. Water Resources Research 17 (3), 686-692.

Cardiff, M., Bakhos, T., Kitanidis, P., Barrash, W., 2013a. Aquifer heterogeneity characterization with oscillatory pumping: Sensitivity analysis and imaging potential. Water Resources Research 49 (9), 5395-5410.

Cardiff, M., Barrash, W., 2011. 3-d transient hydraulic tomography in unconfined aquifers with fast drainage response. Water Resources Research $47(12)$.

Cardiff, M., Barrash, W., 2014. Analytical and semi-analytical tools for the design of oscillatory pumping tests. Groundwater.

Cardiff, M., Barrash, W., Kitanidis, P. K., 2012. A field proof-of-concept of aquifer imaging using 3-d transient hydraulic tomography with modular, temporarily-emplaced equipment. Water Resources Research 48 (5).

Cardiff, M., Barrash, W., Kitanidis, P. K., 2013b. Hydraulic conductivity imaging from 3-d transient hydraulic tomography at several pumping/observation densities. Water Resources Research 49 (11), 7311-7326.

Cardiff, M., Barrash, W., Thoma, M., Malama, B., 2011. Information content of slug tests for estimating hydraulic properties in realistic, highconductivity aquifer scenarios. Journal of Hydrology 403 (1), 66-82. 
Carslaw, H., Jaeger, J., 1959. Conduction of Heat in Solids, second ed. Oxford University Press, London.

Cartwright, N., Nielsen, P., Dunn, S., 2003. Water table waves in an unconfined aquifer: Experiments and modeling. Water Resources Research $39(12)$.

Cartwright, N., Nielsen, P., Perrochet, P., 2005. Influence of capillarity on a simple harmonic oscillating water table: Sand column experiments and modeling. Water Resources Research 41 (8).

Clement, W. P., Barrash, W., 2006. Crosshole radar tomography in a fluvial aquifer near Boise, Idaho. Journal of Environmental \& Engineering Geophysics 11 (3), 171-184.

Dagan, G., 1967. A method of determining the permeability and effective porosity of unconfined anisotropie aquifers. Water Resources Research 3 (4), 1059-1071.

Dagan, G., 1986. Statistical theory of groundwater flow and transport: Pore to laboratory, laboratory to formation, and formation to regional scale. Water Resources Research 22 (9S), 120S-134S.

Dagan, G., Lessoff, S., 2011. Flow to partially penetrating wells in unconfined heterogeneous aquifers: Mean head and interpretation of pumping tests. Water Resources Research 47 (6).

Dagan, G., Rabinovich, A., 2014. Oscillatory pumping wells in phreatic, compressible, and homogeneous aquifers. Water Resources Research 50 (8), 7058-7066. 
Fokker, P. A., Borello, E. S., Serazio, C., Verga, F., 2012a. Estimating reservoir heterogeneities from pulse testing. Journal of Petroleum Science and Engineering 86, 15-26.

Fokker, P. A., Renner, J., Verga, F., 2013. Numerical modeling of periodic pumping tests in wells penetrating a heterogeneous aquifer. American Journal of Environmental Sciences 9 (1), 1.

Fokker, P. A., Renner, J., Verga, F., et al., 2012b. Applications of harmonic pulse testing to field cases. In: SPE Europec/EAGE Annual Conference. Society of Petroleum Engineers.

Fokker, P. A., Verga, F., 2011. Application of harmonic pulse testing to water-oil displacement. Journal of Petroleum Science and Engineering 79 (3), 125-134.

Green, W. H., Ampt, G., 1911. Studies on soil physics, 1. the flow of air and water through soils. J. Agric. Sci 4 (1), 1-24.

Guiltinan, E., Becker, M. W., 2015. Measuring well hydraulic connectivity in fractured bedrock using periodic slug tests. Journal of Hydrology 521, $100-107$.

Hollaender, F., Hammond, P. S., Gringarten, A. C., et al., 2002. Harmonic testing for continuous well and reservoir monitoring. In: SPE Annual Technical Conference and Exhibition. Society of Petroleum Engineers.

Johnson, C. R., Greenkorn, R., Woods, E., et al., 1966. Pulse-testing: a new method for describing reservoir flow properties between wells. Journal of Petroleum Technology 18 (12), 1-599. 
Kuo, C., et al., 1972. Determination of reservoir properties from sinusoidal and multirate flow tests in one or more wells. Society of Petroleum Engineers Journal 12 (06), 499-507.

Mathias, S., Butler, A., 2006. Linearized Richards' equation approach to pumping test analysis in compressible aquifers. Water Resources Research $42(6)$.

Mishra, P. K., Vessilinov, V., Gupta, H., 2013. On simulation and analysis of variable-rate pumping tests. Groundwater 51 (3), 469-473.

Moench, A. F., 2004. Importance of the vadose zone in analyses of unconfined aquifer tests. Groundwater 42 (2), 223-233.

Moret, G. J., Knoll, M. D., Barrash, W., Clement, W. P., 2006. Investigating the stratigraphy of an alluvial aquifer using crosswell seismic traveltime tomography. Geophysics 71 (3), B63-B73.

Mwenifumbo, C. J., Barrash, W., Knoll, M. D., 2009. Capacitive conductivity logging and electrical stratigraphy in a high-resistivity aquifer, Boise Hydrogeophysical Research Site. Geophysics 74 (3), E125-E133.

Nielsen, P., Perrochet, P., 2000. Watertable dynamics under capillary fringes: experiments and modelling. Advances in Water Resources 23 (5), 503-515.

Oppenheim, A. V., Schafer, R. W., Buck, J. R., et al., 1989. Discrete-time signal processing. Vol. 2. Prentice-hall Englewood Cliffs.

Rabinovich, A., 2015. Heat conduction in a semi-infinite medium with time- 
periodic boundary temperature and a circular inhomogeneity. International Journal of Thermal Sciences 87, 146-157.

Rabinovich, A., Dagan, G., Miloh, T., 2012. Heat conduction in a semiinfinite medium with a spherical inhomogeneity and time-periodic boundary temperature. International Journal of Heat and Mass Transfer 55 (4), $618-628$.

Rabinovich, A., Dagan, G., Miloh, T., 2013a. Dynamic effective properties of heterogeneous geological formations with spherical inclusions under periodic time variations. Geophysical Research Letters 40 (7), 1345-1350.

Rabinovich, A., Dagan, G., Miloh, T., 2013b. Effective conductivity of heterogeneous aquifers in unsteady periodic flow. Advances in Water Resources $62,317-326$.

Rasmussen, T. C., Haborak, K. G., Young, M. H., 2003. Estimating aquifer hydraulic properties using sinusoidal pumping at the Savannah River Site, South Carolina, USA. Hydrogeology Journal 11 (4), 466-482.

Renard, P., De Marsily, G., 1997. Calculating equivalent permeability: a review. Advances in Water Resources 20 (5), 253-278.

Renner, J., Messar, M., 2006. Periodic pumping tests. Geophysical Journal International 167 (1), 479-493.

Smeulders, D., Eggels, R., Van Dongen, M., 1992. Dynamic permeability: reformulation of theory and new experimental and numerical data. Journal of Fluid Mechanics 245, 211-227. 
Song, I., Renner, J., 2007. Analysis of oscillatory fluid flow through rock samples. Geophysical Journal International 170 (1), 195-204.

Tartakovsky, G. D., Neuman, S. P., 2007. Three-dimensional saturatedunsaturated flow with axial symmetry to a partially penetrating well in a compressible unconfined aquifer. Water Resources Research 43 (1).

Thoma, M. J., Barrash, W., Cardiff, M., Bradford, J., Mead, J., 2014. Estimating unsaturated hydraulic functions for coarse sediment from a fieldscale infiltration experiment. Vadose Zone Journal 13 (3).

Zhao, S., Wang, F., Xu, H., Zhu, J., 2009. Multi-frequency identification method in signal processing. Digital Signal Processing 19 (4), 555-566. 


\section{List of Figures}

1 Map showing location of the Boise Hydrological Research Site relative to Boise River. Site coordinates: $43^{\circ} 32^{\prime} 33.5^{\prime \prime} \mathrm{N}, 116^{\circ}$ $5,50.5^{\prime \prime} \mathrm{W}$. Inset shows layout of wells in the central area of the site (figure from Cardiff et al. (2012)). . . . . . . . . . . . 45

2 The setup of the pumping test at the BHRS. Pumping is conducted in Well B3 and observation points are in Wells B1, B4 and C4. . . . . . . . . . . . . . . . . 46

3 (a) Oscillating signal generator surface configuration and components: 1. Fly wheel to drive piston and provide sinusoidal signal. Range of attachment positions for adjusting driving piston stroke length (i.e., injection or withdrawal volume); 2. Chamber for driving piston; 3. Hydraulic hoses to actuate downhole piston movement (hydraulic fluid is water); 4. Motor (5 hp, DC); 5. Manual control for flywheel rotation rate (i.e., oscillating signal frequency). (b) Oscillating signal generator downhole components labeled in order of insertion into a well. Tubing for packer inflation and for measurement of hydraulic head below, within, and above the zone of oscillating injection/withdrawal are not shown: 1. Straddle packer below zone of oscillating injection/withdrawal; 2a. Zone of oscillating injection/withdrawal; 2b. Straddle packer above zone of oscillating injection/withdrawal. Riser inside packer is open to piston chamber above and injection/withdrawal zone below; 3a. Piston chamber; 3b. Sealed piston actuation chamber; 4. Hydraulic hoses connecting downhole piston actuation to surface driving piston. . . . . . . . . . . . . . . . . . 47

4 Field test results for (a) hydraulic head as a function of time and (b) amplitude as a function of frequency in the Fourier domain (inset is a log-log plot). The results are for 0717 Test 8 measured in the pumping zone in Well B3 elevation $842.5 \mathrm{~m} .48$

5 Head amplitude (a) and phase (b) as a function of elevation in each of the three observation boreholes and for three different periods of oscillation. The reference phase is taken from Well

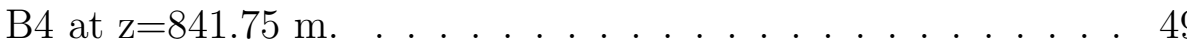


6 The non-dimensional head amplitude as a function of period at Well B4, $\mathrm{z}=837.7 \mathrm{~m}$. Inset: The solution for a point source of oscillating discharge (Eq. (A3)). . . . . . . . . . . . . . 50

7 The equivalent conductivity $K_{e q}$ (a) and specific storage $S_{s, e q}$ (b) as a function of oscillation period. Results obtained using both the confined and unconfined aquifer models are shown as indicated in the legend. . . . . . . . . . . . . . . . . 51

8 Equivalent specific yield as a function of period $P$. Results are obtained using the unconfined aquifer solution, Eq. (A1). . 52

9 A problem of a single spherical inclusion of properties $\alpha_{i n}, K_{\text {in }}$ and $S_{s, i n}$ in a medium of properties $\alpha_{0}, K_{0}$ and $S_{s, 0}$ below a planar boundary of oscillating head. A square box region of side $L_{b}$ is shown in dashed lines. . . . . . . . . . . . . . . . . . 53

10 Equivalent conductivity (a) and the ratio $C=\left(J_{i n}^{2} / J_{i n}^{1}\right) /\left(J_{\text {ex }}^{2} / J_{\text {ex }}^{1}\right)$ (b) as a function of dimensionless period for the problem of a single spherical inhomogeneity (Fig. 9). . . . . . . . . . . . 54

11 Specific yield as a function of dimensionless period. Field test results for $S_{y, e q}$ are compared with previous theoretical and empirical results. . . . . . . . . . . . . . . . . . . 55 


\section{FIGURES}

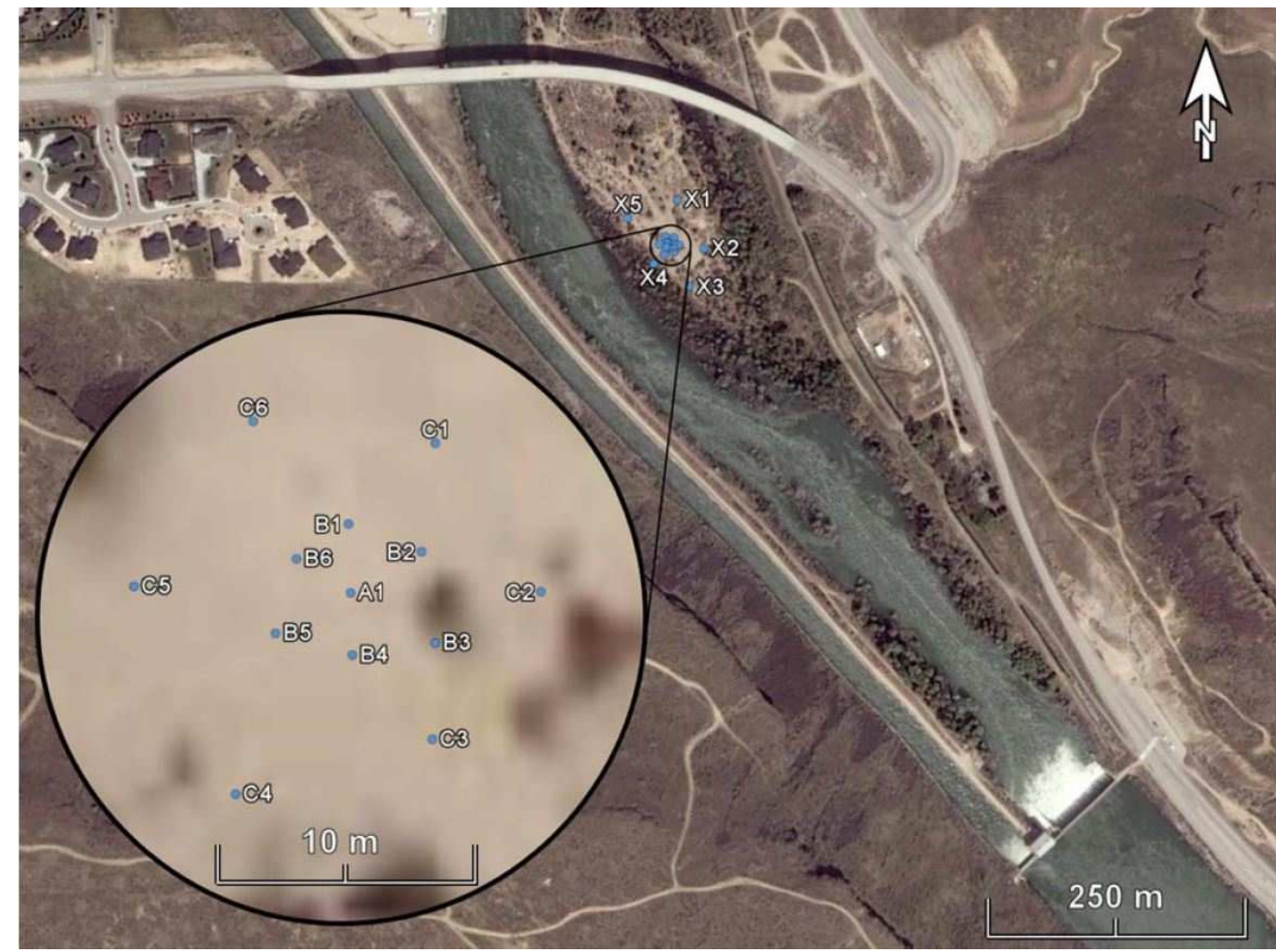

Figure 1: Map showing location of the Boise Hydrological Research Site relative to Boise River. Site coordinates: $43^{\circ} 32^{\prime} 33.5^{\prime \prime} \mathrm{N}, 116^{\circ} 5{ }^{\prime} 50.5^{\prime} \mathrm{W}$. Inset shows layout of wells in the central area of the site (figure from Cardiff et al. (2012)). 


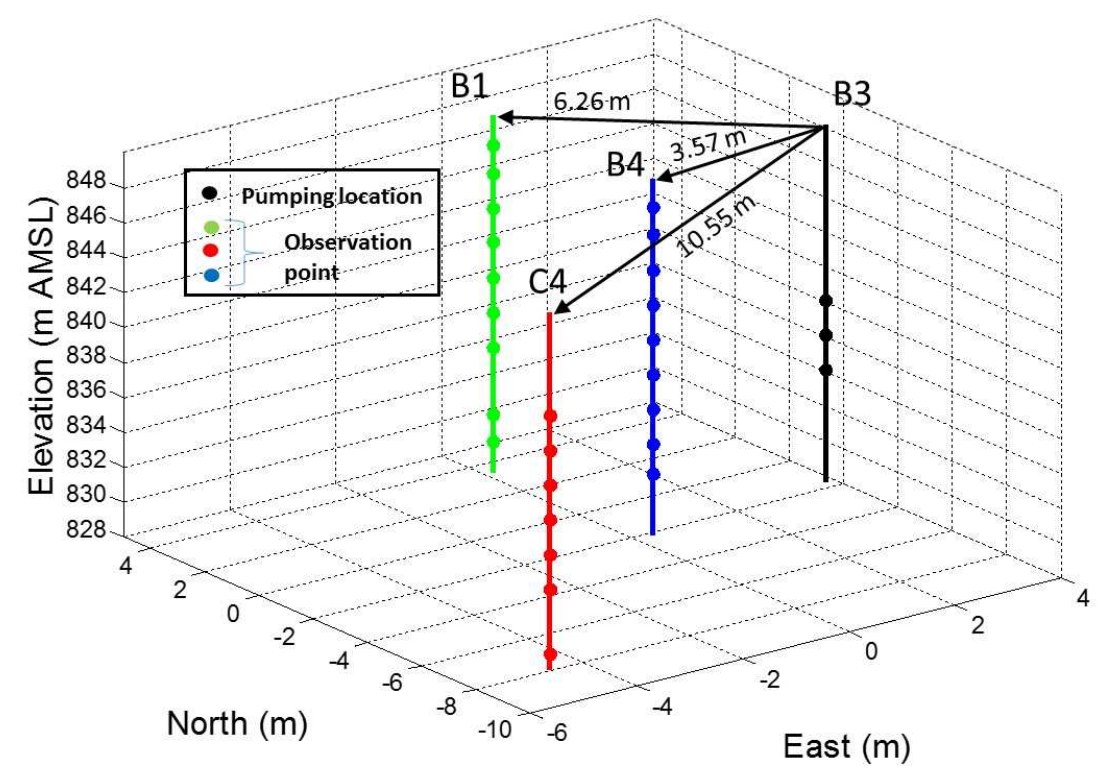

Figure 2: The setup of the pumping test at the BHRS. Pumping is conducted in Well B3 and observation points are in Wells B1, B4 and C4. 


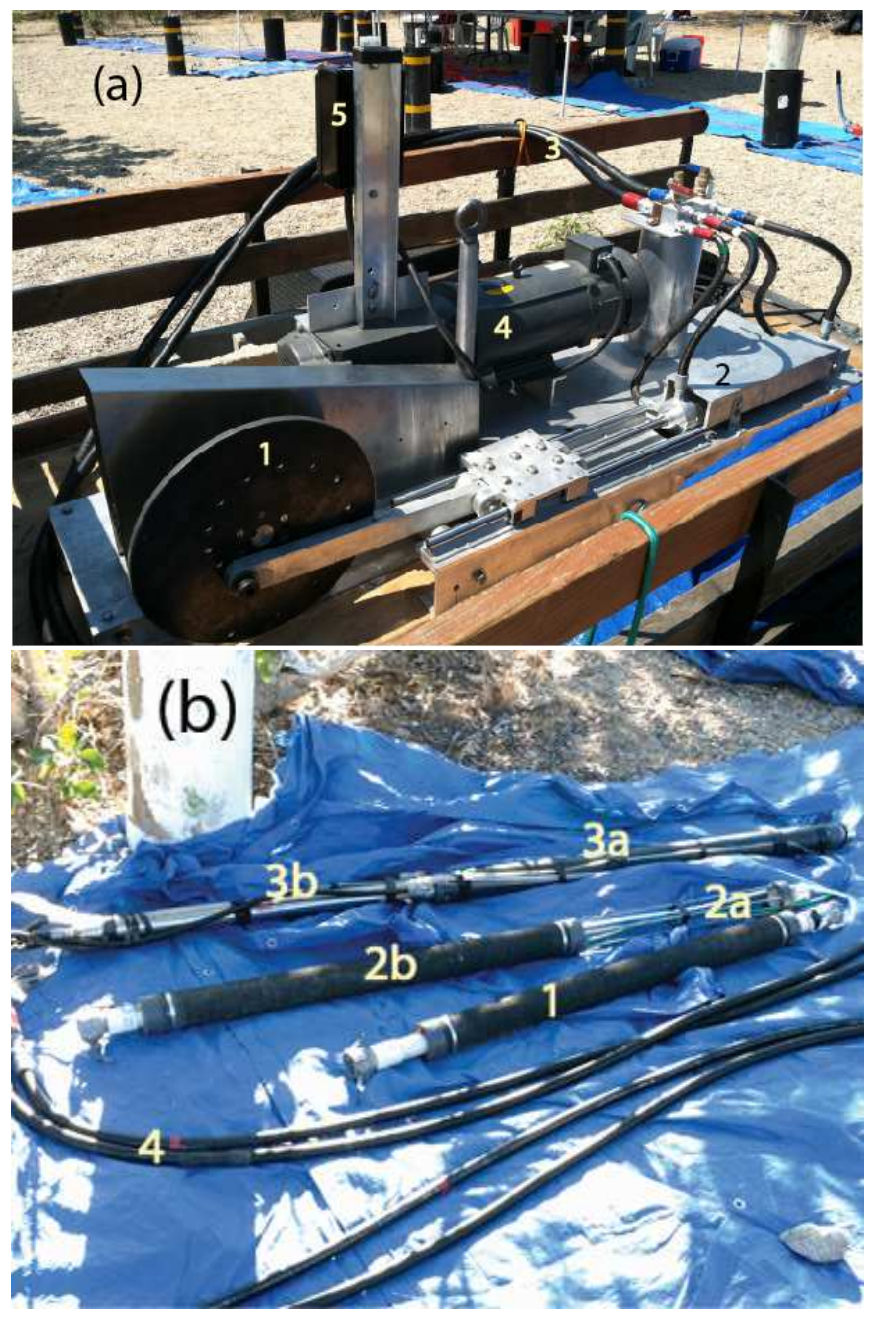

Figure 3: (a) Oscillating signal generator surface configuration and components: 1. Fly wheel to drive piston and provide sinusoidal signal. Range of attachment positions for adjusting driving piston stroke length (i.e., injection or withdrawal volume); 2. Chamber for driving piston; 3 . Hydraulic hoses to actuate downhole piston movement (hydraulic fluid is water); 4. Motor (5 hp, DC); 5. Manual control for flywheel rotation rate (i.e., oscillating signal frequency). (b) Oscillating signal generator downhole components labeled in order of insertion into a well. Tubing for packer inflation and for measurement of hydraulic head below, within, and above the zone of oscillating injection/withdrawal are not shown: 1. Straddle packer below zone of oscillating injection/withdrawal; 2a. Zone of oscillating injection/withdrawal; 2b. Straddle packer above zone of oscillating injection/withdrawal. Riser inside packer is open to piston chamber above and injection/withdrawal zone below; 3a. Piston chamber; 3b. Sealed piston actuation chamber; 4. Hydraulic hoses connecting downhole piston actuation to surface driving piston. 


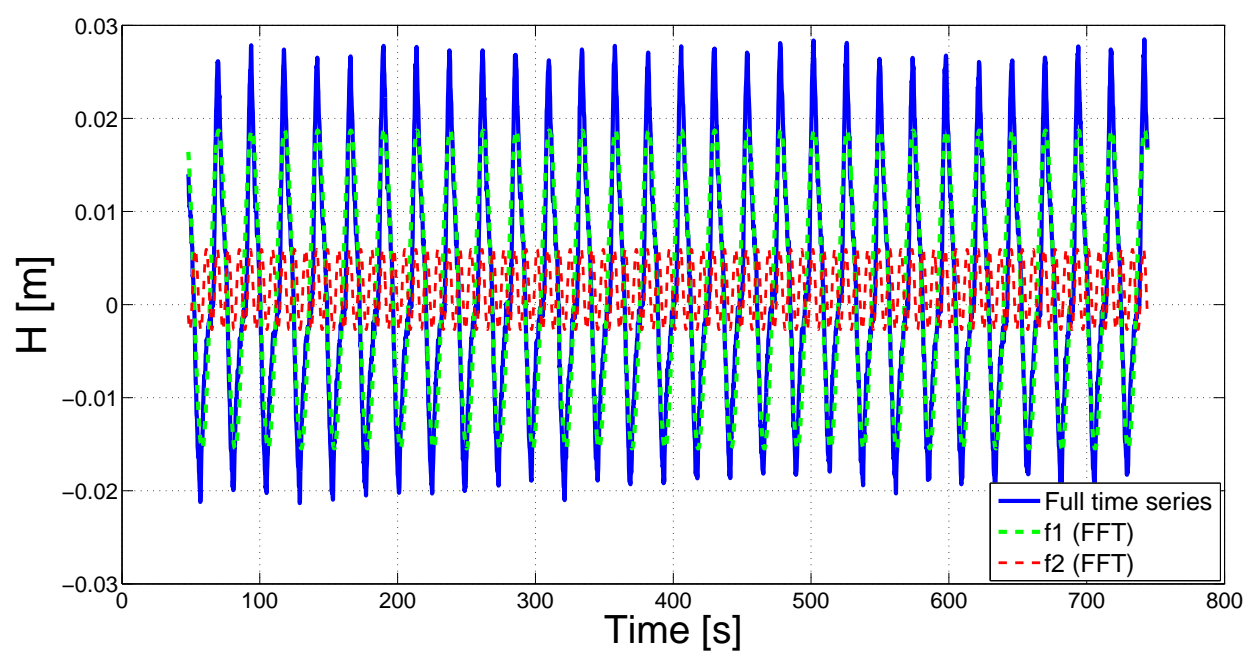

(a)

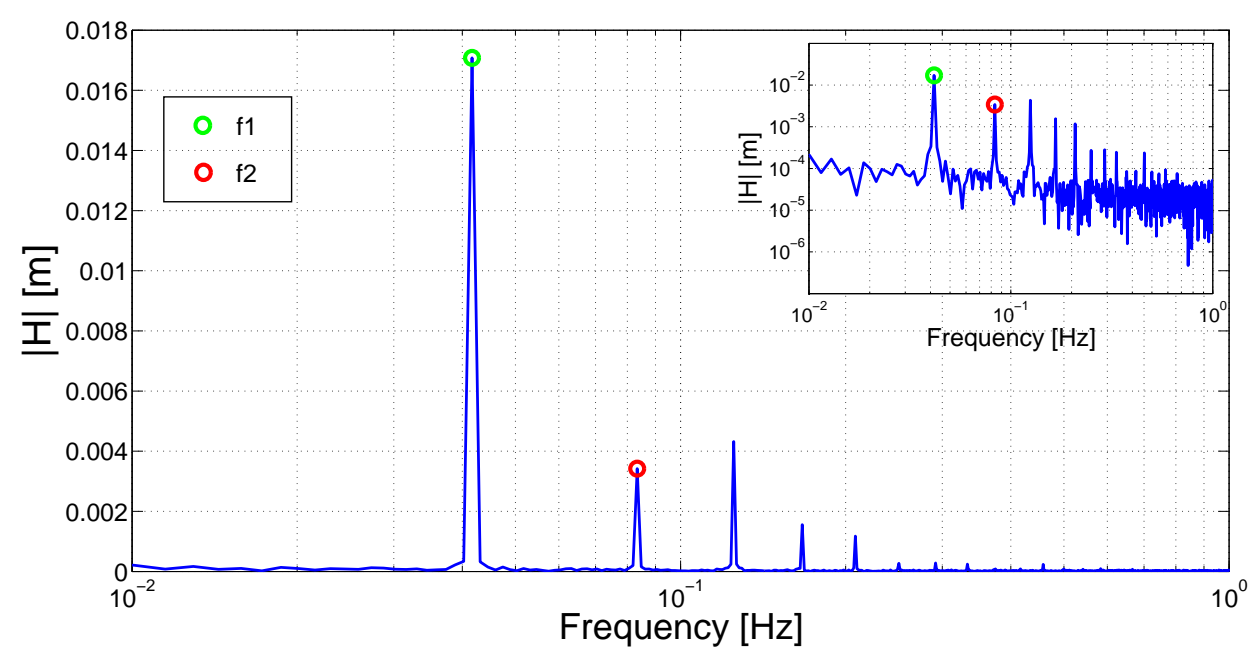

(b)

Figure 4: Field test results for (a) hydraulic head as a function of time and (b) amplitude as a function of frequency in the Fourier domain (inset is a log-log plot). The results are for 0717 Test 8 measured in the pumping zone in Well B3 elevation $842.5 \mathrm{~m}$. 


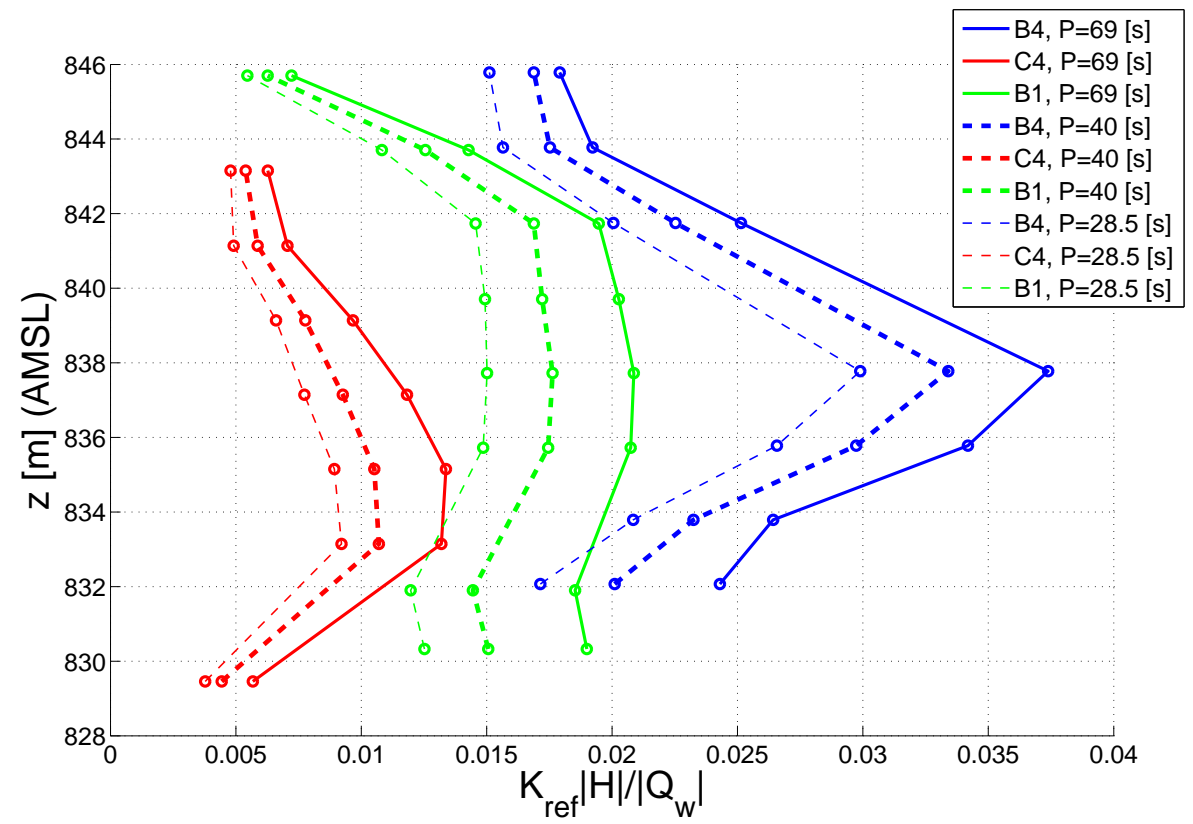

(a)

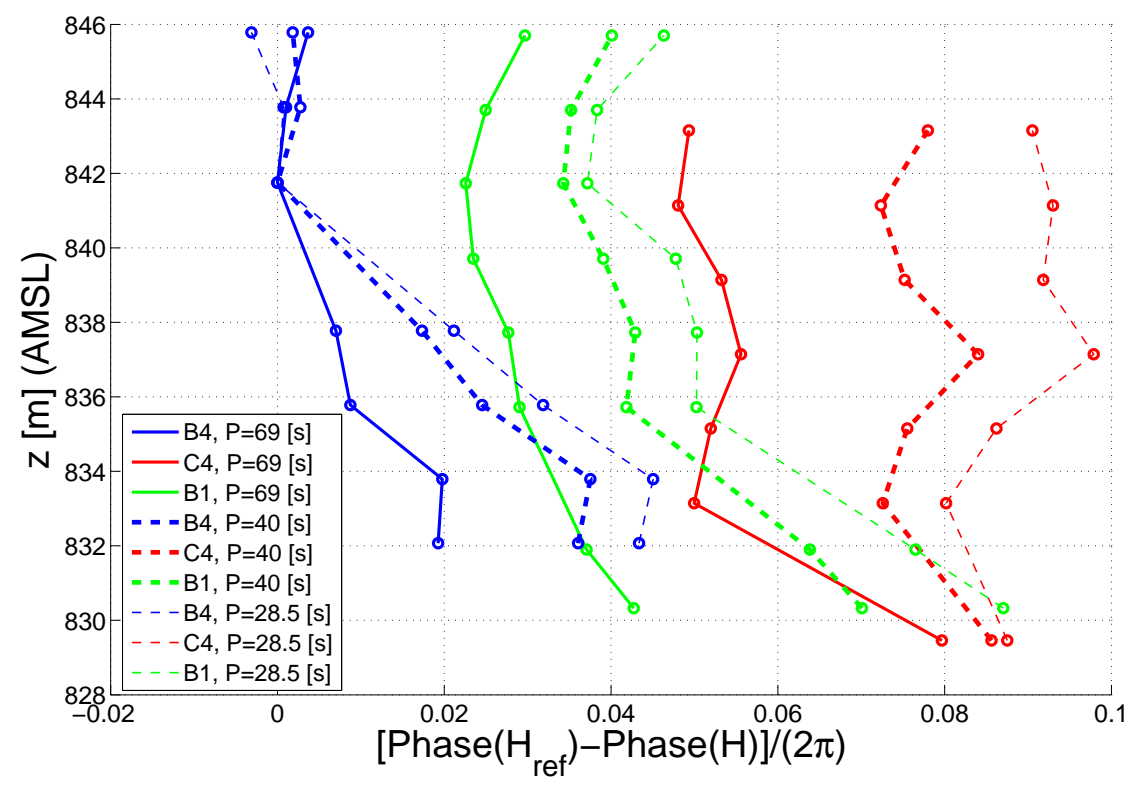

(b)

Figure 5: Head amplitude (a) and phase (b) as a function of elevation in each of the three observation boreholes and for three different periods of oscillation. The reference phase is taken from Well B4 at $\mathrm{z}=841.75 \mathrm{~m}$. 


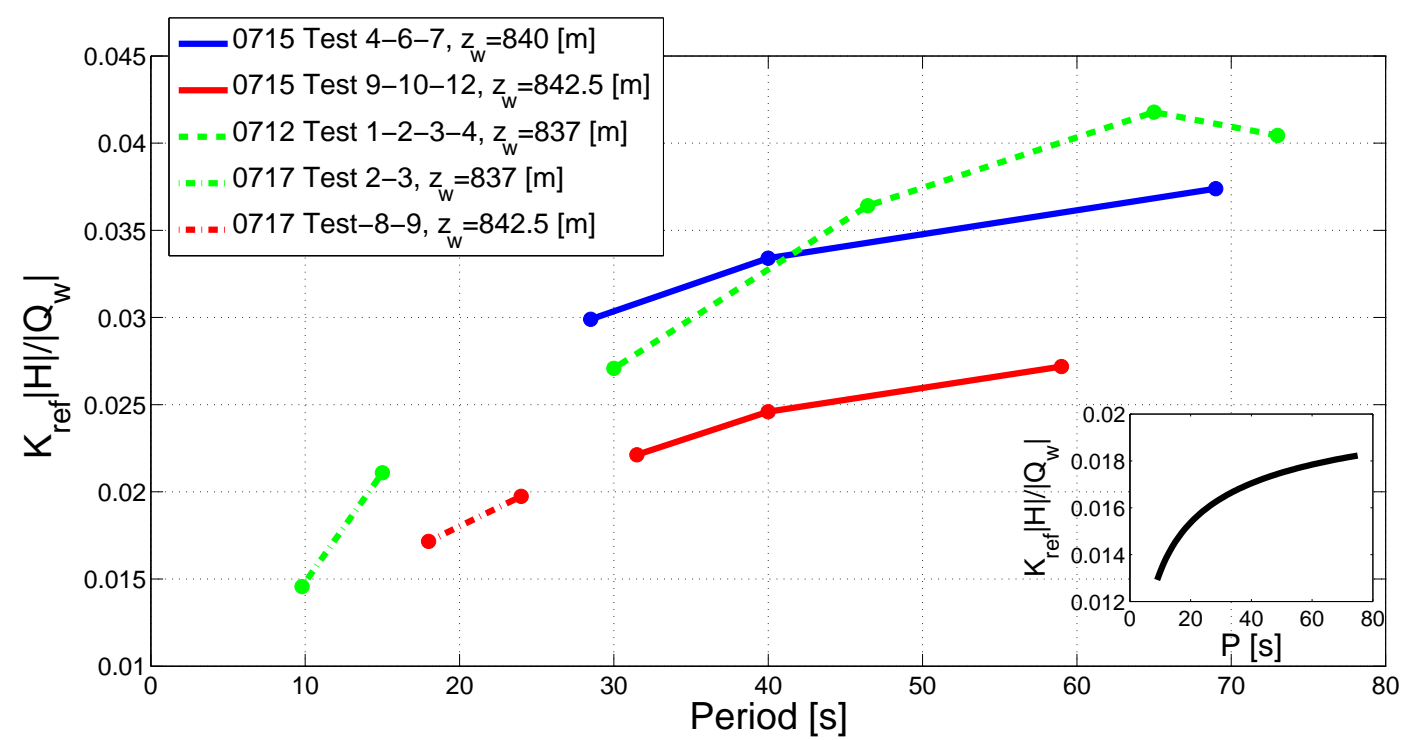

Figure 6: The non-dimensional head amplitude as a function of period at Well $\mathrm{B} 4, \mathrm{z}=837.7 \mathrm{~m}$. Inset: The solution for a point source of oscillating discharge (Eq. (A3)). 


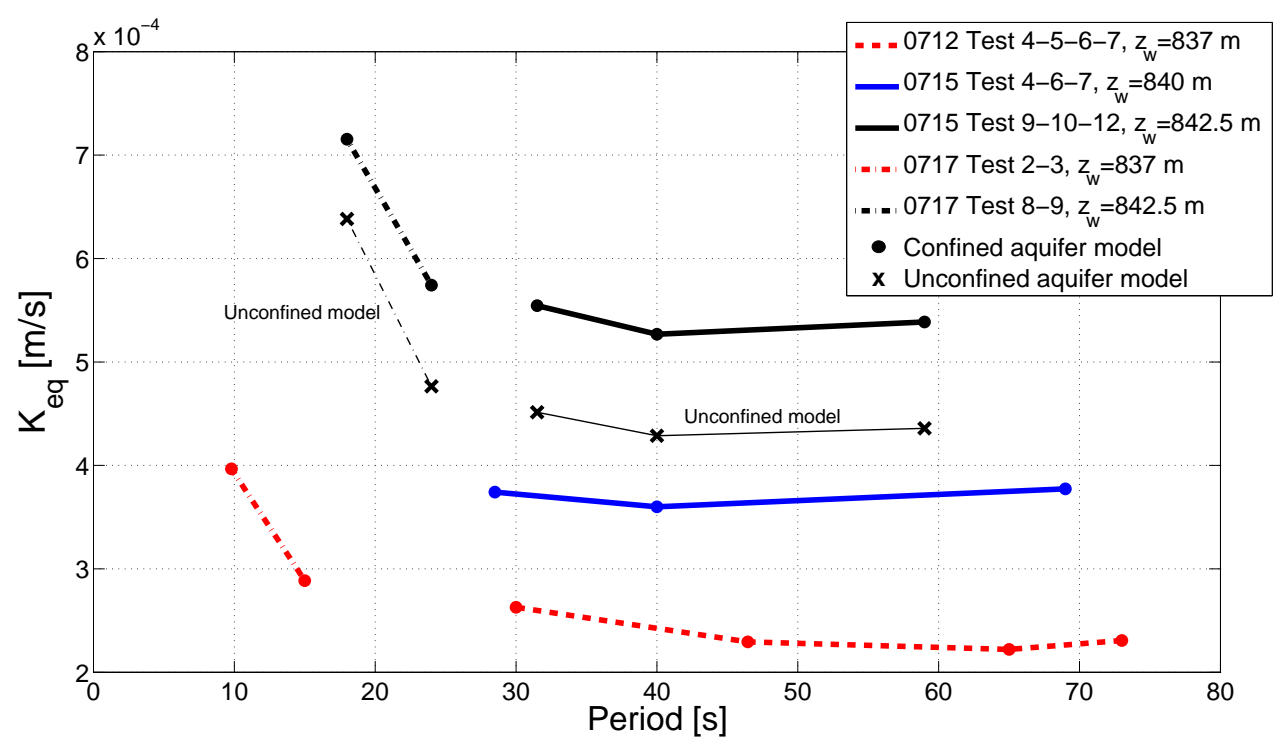

(a)

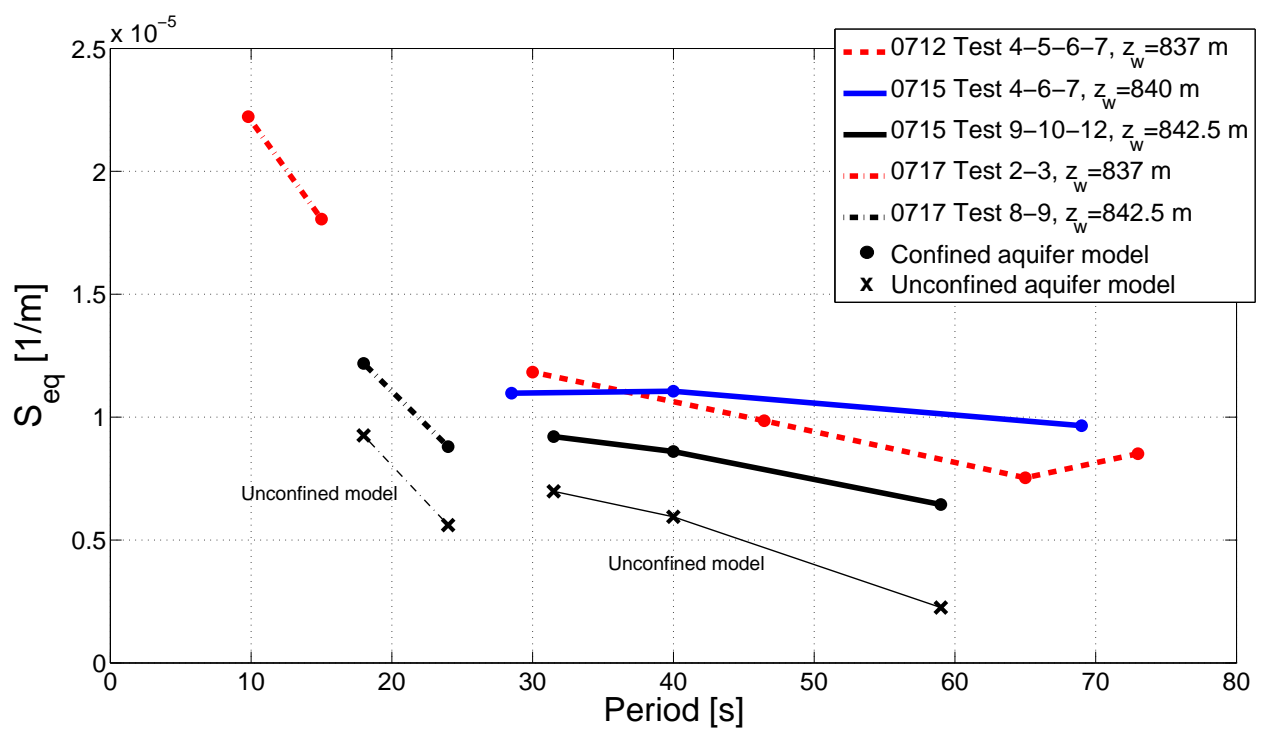

(b)

Figure 7: The equivalent conductivity $K_{e q}$ (a) and specific storage $S_{s, e q}$ (b) as a function of oscillation period. Results obtained using both the confined and unconfined aquifer models are shown as indicated in the legend. 


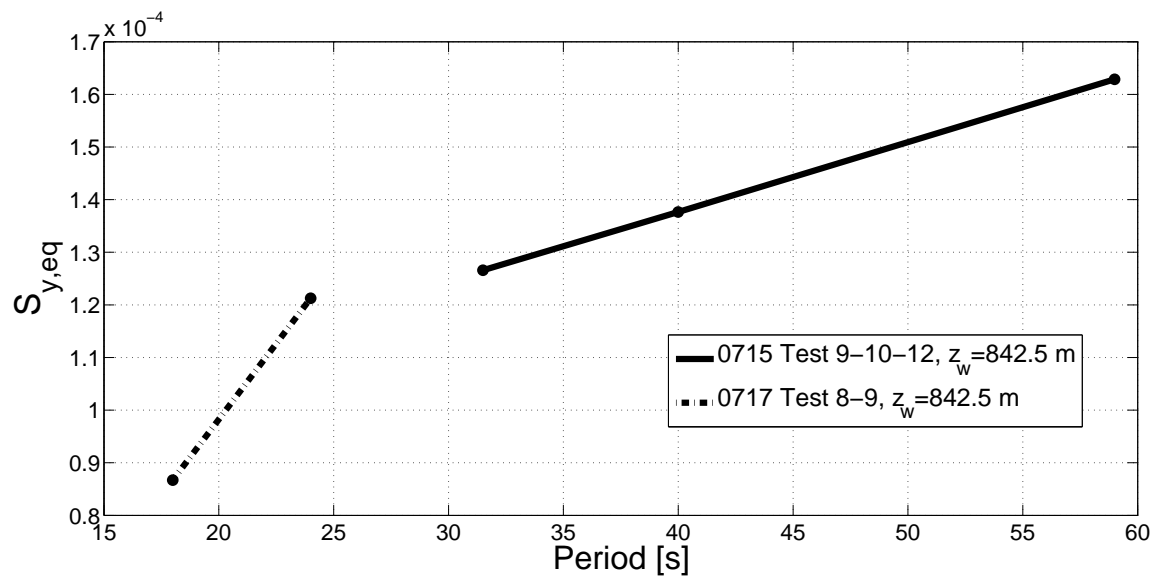

Figure 8: Equivalent specific yield as a function of period $P$. Results are obtained using the unconfined aquifer solution, Eq. (A1). 


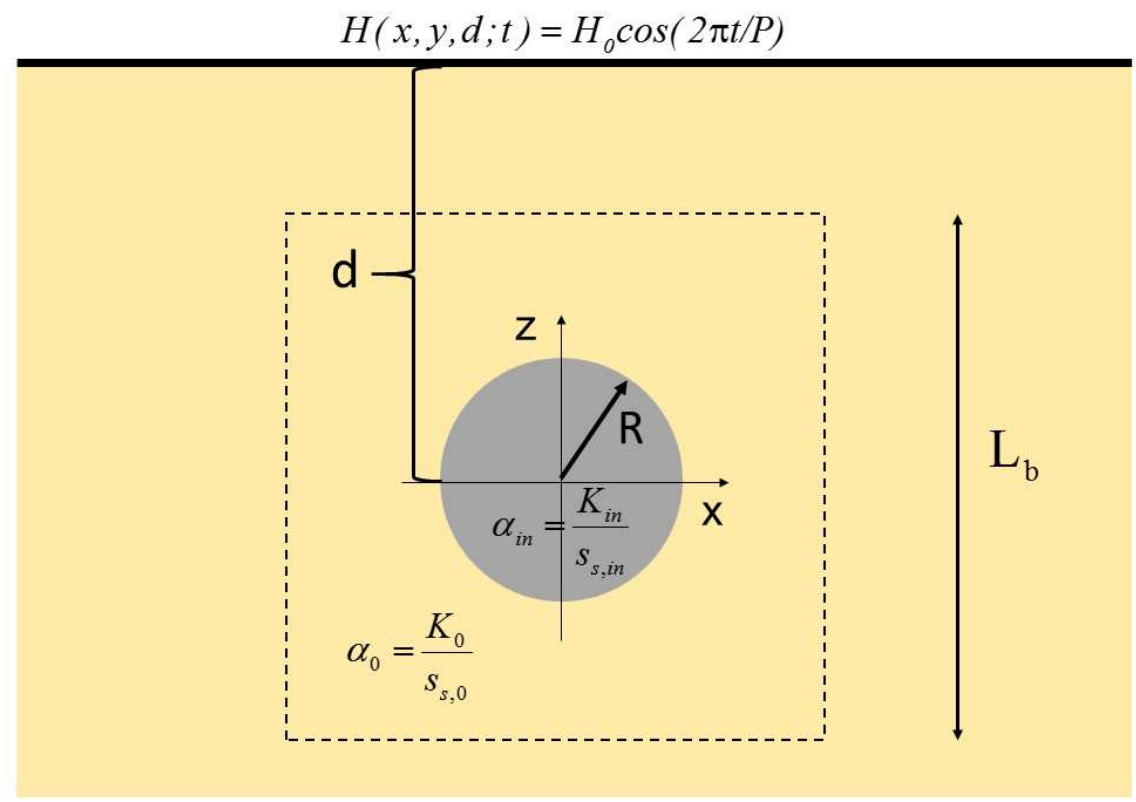

Figure 9: A problem of a single spherical inclusion of properties $\alpha_{i n}, K_{i n}$ and $S_{s, i n}$ in a medium of properties $\alpha_{0}, K_{0}$ and $S_{s, 0}$ below a planar boundary of oscillating head. A square box region of side $L_{b}$ is shown in dashed lines. 


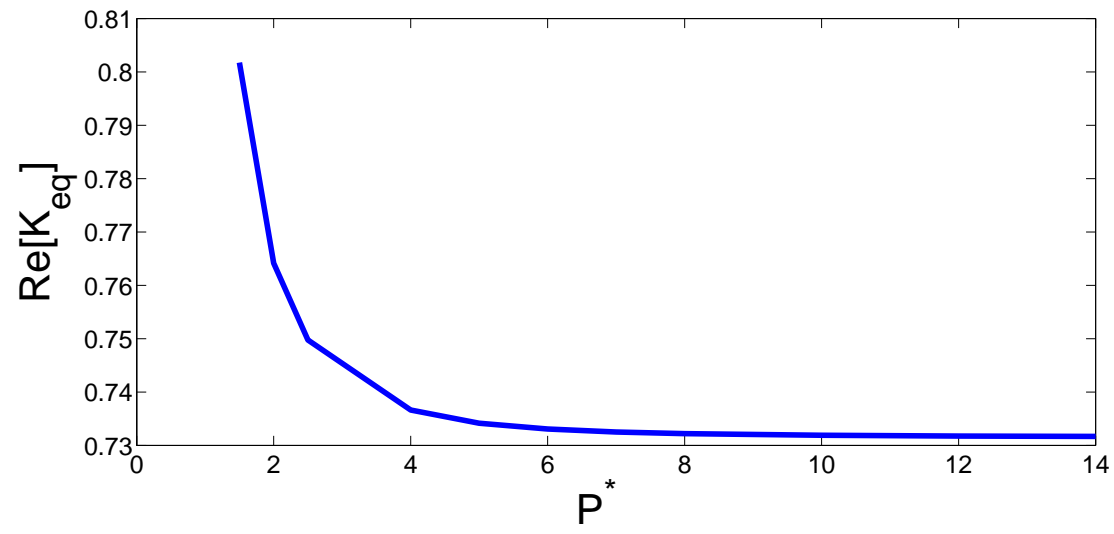

(a)

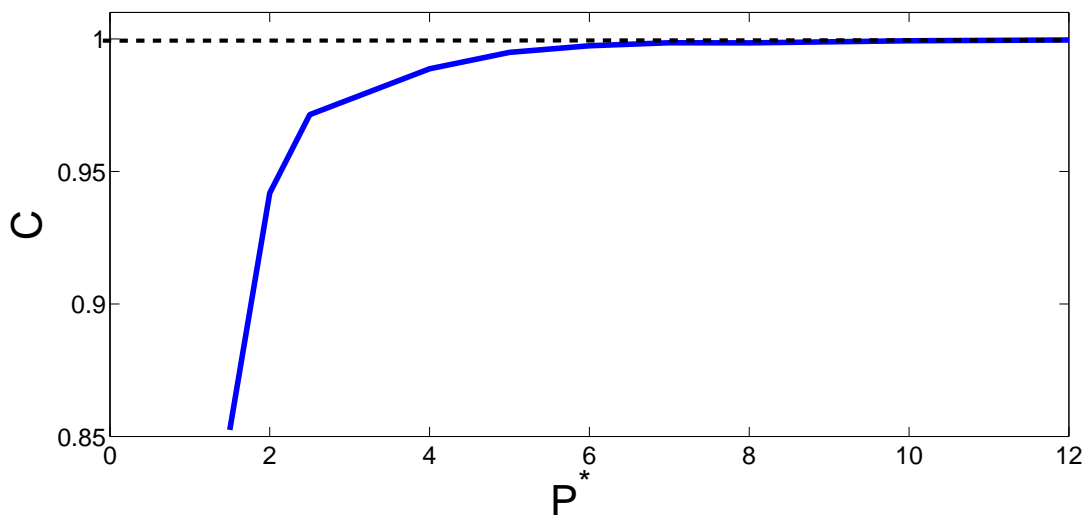

(b)

Figure 10: Equivalent conductivity (a) and the ratio $C=\left(J_{i n}^{2} / J_{i n}^{1}\right) /\left(J_{e x}^{2} / J_{e x}^{1}\right)$ (b) as a function of dimensionless period for the problem of a single spherical inhomogeneity (Fig. 9). 


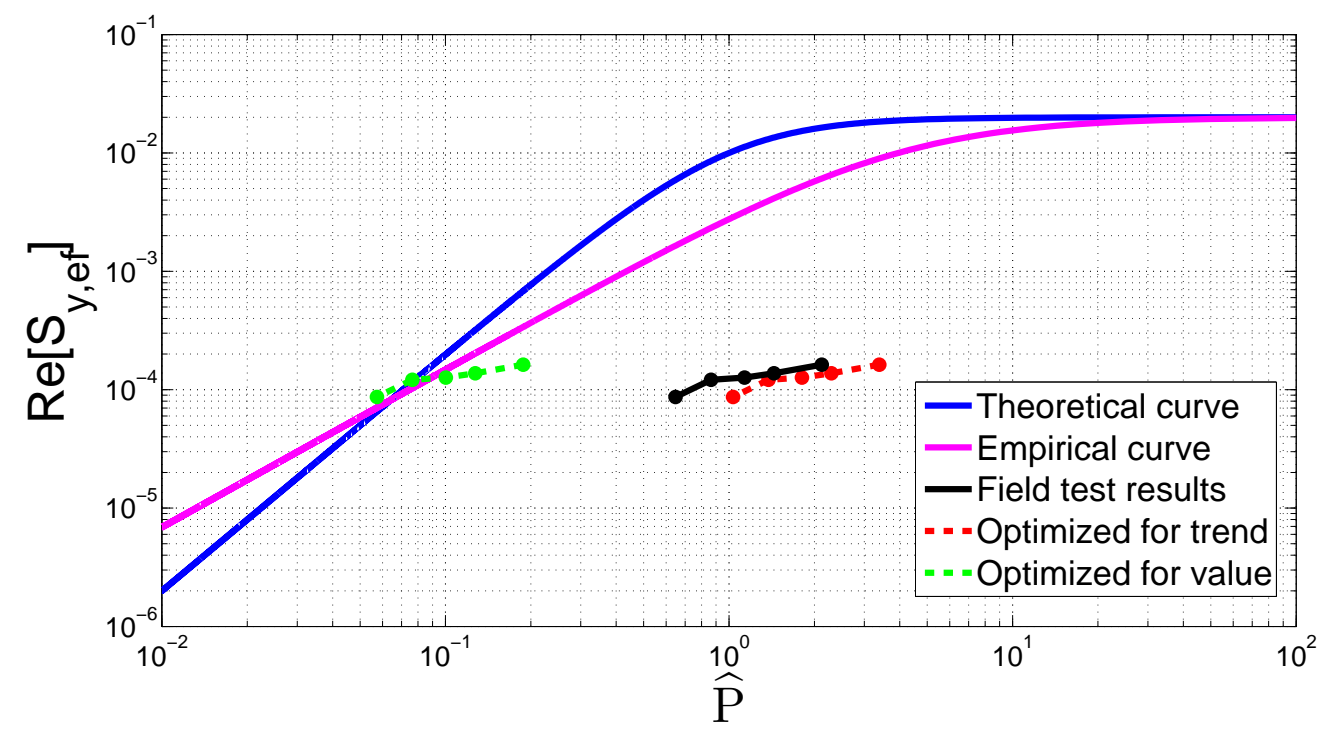

Figure 11: Specific yield as a function of dimensionless period. Field test results for $S_{y, e q}$ are compared with previous theoretical and empirical results. 


\section{List of Tables}

1 Summary of oscillatory tests performed . . . . . . . . . . 57 
Table 1: Summary of oscillatory tests performed

\begin{tabular}{|l|l|l|l|l|}
\hline Test name & $\begin{array}{l}\text { Pumping In- } \\
\text { terval Eleva- } \\
\text { tion }[\mathrm{m}]\end{array}$ & Period $[\mathrm{s}]$ & $\begin{array}{l}\text { Stroke } \\
\text { length }[\mathrm{m}]\end{array}$ & $\begin{array}{l}\text { Discharge ampli- } \\
\text { tude }[\text { liter } / \mathrm{s}]\end{array}$ \\
\hline 0712 Test 1 & $836-837$ & 73 & 0.7 & 0.06 \\
0712 Test 2 & $836-837$ & 46.5 & 0.7 & 0.095 \\
0712 Test 3 & $836-837$ & 65 & 0.7 & 0.068 \\
0712 Test 4 & $836-837$ & 30 & 0.7 & 0.147 \\
0715 Test 4 & $839-840$ & 69 & 0.7 & 0.064 \\
0715 Test 6 & $839-840$ & 40 & 0.7 & 0.11 \\
0715 Test 7 & $839-840$ & 28.5 & 0.7 & 0.154 \\
0715 Test 9 & $839-842.5$ & 59 & 0.7 & 0.075 \\
0715 Test 10 & $841.5-842.5$ & 40 & 0.7 & 0.11 \\
0715 Test 12 & $841.5-842.5$ & 31.5 & 0.7 & 0.14 \\
0717 Test 2 & $836-837$ & 15 & 0.22 & 0.092 \\
0717 Test 3 & $836-837$ & 9.8 & 0.22 & 0.141 \\
0717 Test 8 & $841.5-842.5$ & 24 & 0.22 & 0.058 \\
0717 Test 9 & $841.5-842.5$ & 18 & 0.22 & 0.077 \\
\hline
\end{tabular}

